\title{
Supply-demand diagrams and a new framework for analyzing the inhomogeneous Lighthill-Whitham-Richards model
}

\author{
Wen-Long Jin, University of California, Irvine, CA; Liang Chen, University of \\ Science and Technology of China, Hefei, China; Elbridge Gerry Puckett, \\ University of California, Davis, CA
}

\begin{abstract}
Traditionally, the Lighthill-Whitham-Richards (LWR) models for homogeneous and inhomogeneous roads have been analyzed in flux-density space with the fundamental diagram of the flux-density relation. In this paper, we present a new framework for analyzing the LWR model, especially the Riemann problem at a linear boundary in which the upstream and downstream links are homogeneous and initially carry uniform traffic. We first review the definitions of local supply and demand functions and then introduce the so-called supply-demand diagram, on which a traffic state can be represented by its supply and demand, rather than as density and flux as on a fundamental diagram. It is well-known that the solutions to the Riemann problem at each link are self-similar with a stationary state, and that the wave on the link is determined by the stationary state and the initial state. In our new framework, there can also exist an interior state next to the linear boundary on each link, which takes infinitesimal space, and admissible conditions for the upstream and downstream stationary and interior states can be derived in supply-demand space. With an entropy condition consistent with a local supply-demand method in interior states, we show that the stationary states exist and are unique within the solution framework. We also develop a graphical scheme for solving the Riemann problem, and the results are shown to be consistent with those in the literature. We further discuss asymptotic stationary states on an inhomogeneous ring road with arbitrary initial conditions and demonstrate the existence of interior states with a numerical example. The framework developed in this study is simpler than existing ones and can be extended for analyzing the traffic dynamics in general road networks.
\end{abstract}

\section{Introduction}

Essential to effective and efficient transportation control, management, and planning strategies is a better understanding of the evolution of traffic dynamics on a road network; i.e., the formation, propagation, and dissipation of traffic queues. The 
seminal work by (Lighthill and Whitham 1955) and (Richards 1956) (LWR) attempts to study traffic dynamics with respect to aggregate values such as density $\rho$, speed $v$, and flux $q$. Based on a continuous version of traffic conservation

$$
\frac{\partial \rho}{\partial t}+\frac{\partial q}{\partial x}=0
$$

and an assumption about the fundamental diagram of the flux-density relation $q=$ $Q(\rho)$, the LWR model of a homogeneous road link can be written as

$$
\frac{\partial \rho}{\partial t}+\frac{\partial Q(\rho)}{\partial x}=0
$$

The corresponding speed-density relation is $v=V(\rho) \equiv Q(\rho) / \rho$. Here the maximum or jam density is denoted by $\rho_{j}$; i.e., $\rho \in\left[0, \rho_{j}\right]$. Usually, $V(\rho)$ is a nonincreasing function of traffic density, $v_{f}=V(0)$ is the free flow speed, $V\left(\rho_{j}\right)=0$, and $q=Q(\rho)$ is unimodal with maximum flux or capacity $C=Q\left(\rho_{c}\right)$ where $\rho_{c}$ is the critical density. Finally, traffic states with density higher than $\rho_{c}$ are congested or over-critical, and those with density lower than $\rho_{c}$ are free flowing or under-critical.

Compared with microscopic traffic flow models (e.g. Gazis et al. 1961; Nagel and Schreckenberg (1992) the LWR model can be used to analyze traffic evolution at the aggregate level with shock and rarefaction waves. With its analytical power and simplicity, the LWR theory has been extended for studying traffic dynamics in more general transportation networks. For examples, Daganzo (1997) proposed a traffic flow model for freeways with special lanes and high-occupancy vehicles with a tworegime fundamental diagram, and Wong and Wong (2002) proposed a multi-class model for heterogeneous drivers.

In this paper, we are interested in the LWR model for a road with bottlenecks, where traffic characteristics such as free flow speed, jam density, the number of lanes, and capacity may be different for different locations. In other words, the fundamental diagram $q=Q(x, \rho)$ depends on location. Such a road link is called inhomogeneous and the corresponding inhomogeneous LWR model can be written as

$$
\frac{\partial \rho}{\partial t}+\frac{\partial Q(x, \rho)}{\partial x}=0 .
$$

In order to understand the fundamental properties of equation (3), we usually analyze its Riemann problem at $x=0$. Hereafter, we will refer to the upstream branch as link 1, the downstream branch as link 2 , and $x=0$ as a linear boundary. In the Riemann problem, links 1 and 2 are both homogeneous and initially carry uniform traffic. That is,

$$
Q(x, \rho)=\left\{\begin{array}{l}
Q_{1}(\rho), x<0 \\
Q_{2}(\rho), x>0
\end{array}\right.
$$

and 


$$
\rho(x, t=0)=\left\{\begin{array}{l}
\rho_{1}, x<0 \\
\rho_{2}, x>0
\end{array}\right.
$$

Since (Mochon 1987), there have been many analytical and numerical studies related to the inhomogeneous LWR model in the literature. Roughly speaking, there have been two types of methods for solving the Riemann problem of inhomogeneous LWR model ${ }^{1}$. In the first type, the inhomogeneous LWR model can be analyzed as a non-strictly hyperbolic conservation law (Isaacson and Temple]1992, Lin et al. 1995; Jin and Zhang 2003a) or as a hyperbolic conservation law with a discontinuous flux function (Gimse and Risebro 1990; Gimse 1993, Klingenberg and Risebro 1995, Diehl 1995, 1996b, Diehl and Wallin 1996; Diehl 1996a; Zhang and Liu 2003; Burger et al. 2005, 2008), and various numerical methods can be used (Bale et al. 2002; Zhang and Liu 2005a b; Zhang et al. 2006; Herty et al. 2007). In the second type, the self-similar waves of the Riemann solutions are separated into links 1 and 2 by introducing a stationary state for each link, and the wave on each link is determined by a new Riemann problem of the corresponding homogeneous LWR model (Seguin and Vovelle 2003; Garavello et al. 2007). Here the stationary states are subject to admissible conditions as well as certain entropy conditions. This solution framework was first proposed for solving Riemann problems at general junctions with more than one upstream and downstream link (Holden and Risebro 1995, Coclite et al. 2005). In (Seguin and Vovelle 2003), the method was introduced for solving the inhomogeneous LWR model, and the stationary states are solved for a specific example. In (Garavello et al.|2007), a more general approach was proposed for solving the stationary states with a singular map method. However, all these existing methods solve the Riemann problem in flux-density space: the first type of method is tedious due to the need to analyze kinematic waves on both links at the same time, and the second type of method fails to present the entropy condition in a physically meaningful way. In addition, all existing methods do not account for interior states in stationary shock waves (van Leer 1984, Bultelle et al. 1998) and cannot be easily extended for studying traffic dynamics in a road network (Jin 2003). Note that, in this paper, we do not intend to study numerical solution methods for solving the inhomogeneous LWR model.

In this paper, we present a new framework for analyzing the inhomogeneous LWR model. We also adopt the method of wave separation by (Holden and Risebro 1995), but introduce a stationary state and an interior state for each branch. Here stationary states are the self-similar states at the boundary, and interior states do not take any space in the continuous solution and only show up in the numerical solutions as observed in (van Leer 1984; Bultelle et al. 1998). Rather than using the fundamental diagram, we introduce a so-called supply-demand diagram and discuss the problem in supply-demand space. After deriving admissible solutions for upstream and downstream stationary and interior states in supply-demand space, we

${ }^{1}$ In (Daganzo 2006), the inhomogeneous LWR model is solved in the space of cumulative number of vehicles as a calculus of variations problem, and the existence of its solution is proved for road links with point bottlenecks. However, the wave solutions of the Riemann problem are not explicitly discussed. 
introduce an entropy condition based on the discrete supply-demand method (Daganzo 1995a; Lebacque 1996). We then prove that stationary states exist and are unique for given upstream demand and downstream supply, and interior states exist but may not be unique. Further we compare the Riemann solutions obtained by the new method with those in the literature for both the homogeneous and inhomogeneous LWR models. We also apply the new framework for analyzing asymptotic stationary states on an inhomogeneous ring road and demonstrate the existence of interior states with numerical examples.

The rest of the paper is organized as follows. In Section 2, we review the definitions of the supply and demand functions and the discrete supply-demand method for computing boundary fluxes. In Section 3, we introduce the supply-demand diagrams and the structure of the solutions to the Riemann problem of the inhomogeneous LWR model in supply-demand space. In section 4, we derive the admissible conditions for stationary and interior states in supply-demand space and an entropy condition consistent with the local supply-demand method in interior states. In Section 5, we solve the Riemann problem for both the homogeneous and inhomogeneous LWR models and present a graphical solution scheme. In Section 6, we analyze asymptotic stationary states on an inhomogeneous ring road and demonstrate the existence of interior states with numerical solutions. In Section 7, we conclude our study with a discussion of future directions.

\section{Review of the supply-demand functions and methods}

\subsection{Review of Engquist-Osher functions and the Godunov method for convex conservation laws}

For the original LWR model (2), assuming that $k=\rho_{c}-\rho$, we obtain a hyperbolic conservation law in $k \in\left[-\rho_{c}, \rho_{j}-\rho_{c}\right]$ as follows

$$
\frac{\partial k}{\partial t}+\frac{\partial f(k)}{\partial x}=0
$$

where $f(k)=C-Q\left(\rho_{c}-k\right)$ is convex when $Q(\rho)$ is concave, since $\frac{\partial^{2} f(k)}{\partial k^{2}}=$ $-\frac{\partial^{2} Q(\rho)}{\partial \rho^{2}} \geq 0$. Here $f(0)=0$. Moreover, if $q=Q(\rho)$ is the Greenshields fundamental diagram (Greenshields 1935), then (6) is Burgers' equation.

For the nonlinear equation (6), we usually have to resort to numerical solutions for general initial and boundary conditions. After dividing the time duration into a number of time intervals of $\Delta t$ and splitting the road link into a number of cells of width $\Delta x$, the finite difference equation in conservation form can be written as follows (Colella and Puckett 2004, LeVeque 2002): 


$$
k_{i}^{j+1}=k_{i}^{j}-\frac{\Delta t}{\Delta x}\left(f_{i+1 / 2}^{j *}-f_{i-1 / 2}^{j *}\right),
$$

where $k_{i}^{j}$ is the average value of $k(x, t)$ in cell $i$ between $x_{i}-\frac{\Delta x}{2}$ and $x_{i}+\frac{\Delta x}{2}$ at time step $j$, and $f_{i+1 / 2}^{j *}$ is the flux through the boundary $x_{1}+\frac{\Delta x}{2}$ between time steps $j$ and $j+1$. Here $\Delta x$ and $\Delta t$ have to satisfy the so-called CFL condition (Courant et al. 1928).

For a hyperbolic conservation law (6), the following functions were first introduced by (Engquist and Osher 1980a b, 1981; Osher and Solomon|1982)

$$
\begin{aligned}
g(k) & =f(\max \{k, 0\})=\left\{\begin{array}{ll}
f(k), & \text { if } k \geq 0 \\
0, & \text { if } k \leq 0
\end{array},\right. \\
& =\int_{0}^{k} \chi(s) f^{\prime}(s) d s=\int_{0}^{k} \max \left\{f^{\prime}(s), 0\right\} d s, \\
h(k) & =f(\min \{k, 0\})=\left\{\begin{array}{ll}
f(k), & \text { if } k \leq 0 \\
0, & \text { if } k \geq 0
\end{array},\right. \\
& =\int_{0}^{k}(1-\chi(s)) f^{\prime}(s) d s=\int_{0}^{k} \min \left\{f^{\prime}(s), 0\right\} d s,
\end{aligned}
$$

where $\chi(k)$ equals 1 iff $f^{\prime}(k) \geq 0$ and equals 0 otherwise. Note that $f(k)=g(k)+$ $h(k)$ and $f(k)=\max \{g(k), h(k)\}$. Therefore, we can rewrite 6 in the following form:

$$
\begin{aligned}
k_{t}+g(k)_{x}+h(k)_{x} & =0, \\
k_{t}+[\max \{g(k), h(k)\}]_{x} & =0 .
\end{aligned}
$$

Further, based on these definitions, the following E-O flux is introduced (Engquist and Osher 1980b, Osher 1984)

$$
\begin{aligned}
f_{i-1 / 2}^{j *} & =g\left(k_{i-1}^{j}\right)+h\left(k_{i}^{j}\right)=f\left(k_{i-1}^{j}\right)+\int_{k_{i-1}}^{k_{i}} \min \left\{f^{\prime}(s), 0\right\} d s \\
& =f\left(k_{i}^{j}\right)-\int_{k_{i-1}}^{k_{i}} \max \left\{f^{\prime}(s), 0\right\} d s \\
& =\frac{1}{2}\left[f\left(k_{i-1}^{j}\right)+f\left(k_{i}^{j}\right)-\int_{k_{i-1}}^{k_{i}}\left|f^{\prime}(s)\right| d s\right] .
\end{aligned}
$$

That is, (7) can be written as

$$
k_{i}^{j+1}=k_{i}^{j}-\frac{\Delta t}{\Delta x}\left(h\left(k_{i+1}^{j}\right)-h\left(k_{i}^{j}\right)+g\left(k_{i}^{j}\right)-g\left(k_{i-1}^{j}\right)\right) .
$$

This can be considered as upwind method for 10 , since $g(k)$ is non-decreasing and $h(k)$ non-increasing.

In (van Leer 1984), the Godunov flux (Godunov 1959) for Burgers' equation was written as 


$$
f_{i-1 / 2}^{j *}=\max \left\{g\left(k_{i-1}^{j}\right), h\left(k_{i}^{j}\right)\right\} .
$$

In (Osher 1984), a new formulation of the Godunov flux (Godunov 1959) was introduced as

$$
f_{i-1 / 2}^{j *}=\left\{\begin{array}{l}
\min _{k_{i-1}^{j} \leq k \leq k_{i}^{j}} f(k), k_{i-1}^{j} \leq k_{i}^{j}, \\
\max _{k_{i-1}^{j} \geq k \geq k_{i}^{j}} f(k), k_{i-1}^{j}>k_{i}^{j} .
\end{array}\right.
$$

For convex $f(k)=\max \{g(k), h(k)\}$, since $g(k)$ and $h(k)$ are monotonically increasing and decreasing respectively, this is equivalent to

$$
\begin{aligned}
& f_{i-1 / 2}^{j *}=\left\{\begin{array}{l}
\max \left\{\min _{k_{i-1}^{j} \leq k \leq k_{i}^{j}} g(k), \min _{k_{i-1}^{j} \leq k \leq k_{i}^{j}} h(k)\right\}, \quad k_{i-1}^{j} \leq k_{i}^{j} \\
\max \left\{\max _{k_{i-1}^{j} \geq k \geq k_{i}^{j}} g(k), \max _{k_{i-1}^{j} \geq k \geq k_{i}^{j}} h(k)\right\}, k_{i-1}^{j}>k_{i}^{j}
\end{array}\right. \\
& =\max \left\{g\left(k_{i-1}^{j}\right), h\left(k_{i}^{j}\right)\right\} .
\end{aligned}
$$

That is, (15) is equivalent to (14). However, it has been shown that (15) can also be applied to non-convex $f(k)$.

\subsection{Review of supply and demand functions and Godunov methods for the LWR model}

For the LWR model [2], we define the following functions

$$
\begin{aligned}
D(\rho) & =Q\left(\min \left\{\rho, \rho_{c}\right\}\right)=\left\{\begin{array}{ll}
Q(\rho), & \text { if } \rho \leq \rho_{c} \\
C, & \text { if } \rho \geq \rho_{c}
\end{array},\right. \\
& =\int_{0}^{\rho} \chi(s) Q^{\prime}(s) d s=\int_{0}^{\rho} \max \left\{Q^{\prime}(s), 0\right\} d s \\
S(\rho) & =Q\left(\max \left\{\rho, \rho_{c}\right\}\right)=\left\{\begin{array}{ll}
Q(\rho), & \text { if } \rho \geq \rho_{c} \\
C, & \text { if } \rho \leq \rho_{c}
\end{array},\right. \\
& =C+\int_{0}^{\rho}(1-\chi(s)) Q^{\prime}(s) d s=C+\int_{0}^{\rho} \min \left\{Q^{\prime}(s), 0\right\} d s,
\end{aligned}
$$

where $\chi(\rho)$ equals 1 iff $Q^{\prime}(\rho) \geq 0$ and equals 0 otherwise. It is straightforward to show that $D(\rho)=C-g(k)$ and $S(\rho)=C-h(k)$. Therefore, the Godunov method for 2 is equivalent to

$$
\rho_{i}^{j+1}=\rho_{i}^{j}-\frac{\Delta t}{\Delta x}\left(q_{i+1 / 2}^{j *}-q_{i-1 / 2}^{j *}\right),
$$

where the boundary flux can be written as (van Leer 1984)

$$
q_{i-1 / 2}^{j *}=\min \left\{D\left(\rho_{i-1}^{j}\right), S\left(\rho_{i}^{j}\right)\right\},
$$


or as (Osher 1984)

$$
q_{i-1 / 2}^{j *}=\left\{\begin{array}{l}
\min _{\rho_{i-1}^{j} \leq \rho \leq \rho_{i}^{j}} Q(\rho), \rho_{i-1}^{j}<\rho_{i}^{j} \\
\max _{\rho_{i}^{j} \leq \rho \leq \rho_{i-1}^{j}} Q(\rho), \rho_{i}^{j}<\rho_{i-1}^{j}
\end{array} .\right.
$$

From 20, , we can see that 19$]$ is valid as long as the fundamental diagram $Q(\rho)$ is unimodal and may not be concave.

In the transportation literature, (Bui et al. 1992) first applied (18) and 20) for solving the LWR model. In (Daganzo 1995a), a new finite difference form was proposed for the LWR model with a triangular or trapezoidal fundamental diagram

$$
Q(\rho)=\min \left\{v_{f} \rho, v_{c}\left(\rho_{j}-\rho\right), Q_{\max }\right\}
$$

where $v_{c}=v_{f} \frac{\rho_{c}}{\rho_{j}-\rho_{c}}$ is the absolute value of the shock wave speed in congested traffic. In the so-called cell transmission model (CTM), the space-time domain was discretized with a CFL number of 1 ; i.e., $\Delta x=v_{f} \Delta t$, and the boundary flux in (18) was written as

$$
q_{i-1 / 2}^{j *}=\min \left\{\bar{D}\left(\rho_{i-1}^{j}\right), \bar{S}\left(\rho_{i}^{j}\right)\right\}
$$

where $\bar{D}\left(\rho_{i}^{j}\right) \Delta t=\min \left\{Q_{\max } \Delta t, n_{i}^{j}\right\}$ and $\bar{S}\left(\rho_{i}^{j}\right) \Delta t=\min \left\{Q_{\max } \Delta t, \frac{v_{c}}{v_{f}}\left(N_{\max }-n_{i}^{j}\right)\right\}$ are defined as "the maximum flows that can be sent and received by cell $i$ in the interval between time steps $j$ and $j+1$ ", $n_{i}^{j}=\rho_{i}^{j} \Delta x=\rho_{i}^{j} v_{f} \Delta t$ is the number of vehicles in cell $i$ at time step $j$, and $N_{\max }=\rho_{j} \Delta x=\rho_{j} v_{f} \Delta t$ is the maximum number of vehicles in cell $i$. Hence the physical meaning of 22 is that the boundary flux is the minimum of the upstream sending flux and the downstream receiving flux. It can be shown that $\bar{D}(\rho)=D(\rho)$ and $\bar{S}(\rho)=S(\rho)$. Thus, 22 is equivalent to 19. for a CFL number of 1 and triangular or trapezoidal fundamental diagrams.

Following (Lebacque 1996), we refer to $D(\rho)$ in $(16)$ and $S(\rho)$ in $(17)$ as the demand and supply functions respectively and call 190 the discrete supply-demand method for computing fluxes. The physical interpretations of demand and supply functions and the supply-demand method have formed the basis for extending the supply-demand method for computing fluxes through various network junctions (Daganzo 1995a; Lebacque 1996, Jin 2003). For inhomogeneous roads, the extension is straightforward as follows (Daganzo 1995a; Lebacque 1996): the demand and supply functions in 16 and $(17)$ are location-dependent, $D(x, \rho(x, t))=$ $Q\left(x, \min \left\{\rho(x, t), \rho_{c}(x)\right\}\right)$ and $S(x, \rho(x, t))=Q\left(x, \max \left\{\rho(x, t), \rho_{c}(x)\right\}\right)$, and the flux is still computed by the supply-demand method $19,, q_{i-1 / 2}^{j *}=\min \left\{D_{i-1}\left(\rho_{i-1}^{j}\right), S_{i}\left(\rho_{i}^{j}\right)\right\}$, where $D_{i-1}(\rho)$ is the demand function in cell $i-1$, and $S_{i}(\rho)$ is the supply function in cell $i$. It has been shown that the flux by the extended supply-demand method is still the Godunov flux (Jin and Zhang 2003a, Zhang and Liu 2003).

In this study, based on the Godunov finite difference equation in (18) and the supply-demand method in 19 for computing boundary fluxes, we attempt to construct the convergent solution of (3) with discontinuous flux functions (4) and initial 
conditions (5). In (Daganzo 1995b), the convergence, truncation error, and capture of shock waves were directly derived from the corresponding finite difference equation of the homogeneous LWR model. Here, we attempt to present a new framework and find the solutions to the Riemann problem of the inhomogeneous LWR model at a linear boundary.

\section{Supply-demand diagrams and the structure of Riemann solutions}

In the literature, the inhomogeneous LWR model has been analyzed in flux-density space. Since a traffic state in flux-density space $(\rho, q)$ can also be represented in supply-demand space as $U=(D, S)$, in this study we will analyze the inhomogeneous LWR model in supply-demand space. Furthermore, we present the structure of solutions to the Riemann problem of the inhomogeneous LWR model in supplydemand space.

\subsection{Supply-demand diagrams}

Corresponding to the fundamental diagram in flux-density space, a supply-demand diagram can be introduced in supply-demand space. In Figure 1 (b), we draw a supply-demand diagram for the two fundamental diagrams in Figure 1(a). On the dashed branch of the supply-demand diagram, traffic is under-critical (UC) and $U=(D, C)$ with $D \leq C$; on the solid branch, traffic is over-critical (OC) and $U=(C, S)$ with $S \leq C$. Compared with the fundamental diagram of a road section, the supply-demand diagram only considers capacity $C$ and congestion level of traffic flow, but not other detailed characteristics such as critical density, jam density, or relationship between density and flux. That is, different fundamental diagrams can have the same supply-demand diagram, as long as they have the same capacity and are unimodal, and their critical densities, jam densities, or shapes are not relevant. However, there is always a one-to-one mapping between a given supply-demand diagram and its corresponding fundamental diagram. That is, there exists a one-to-one mapping between $(\rho, q)$ and $(D, S)$.

For the demand and supply functions in (16) and (17), we can see that $D$ is nondecreasing with $\rho$ and $S$ non-increasing. Thus $D \leq C$, and $S \leq C$, and $\max \{D, S\}=$ $C$. In addition, $D=S=C$ iff traffic is critical; $D<S=C$ iff traffic is strictly undercritical (SUC); $S<D=C$ iff traffic is strictly over-critical (SOC). Therefore, the state $U=(D, S)$ is under-critical (UC), iff $S=C$, or equivalently $D \leq S$. The state $U=(D, S)$ is over-critical (OC), iff $D=C$, or equivalently $S \leq D$.

From a state on the supply-demand diagram, we can obtain the corresponding flux $q(U)=\min \{D, S\}$ and capacity $C=\max \{D, S\}$. However, we cannot tell the density from the supply-demand diagram, and the fundamental diagram is still 


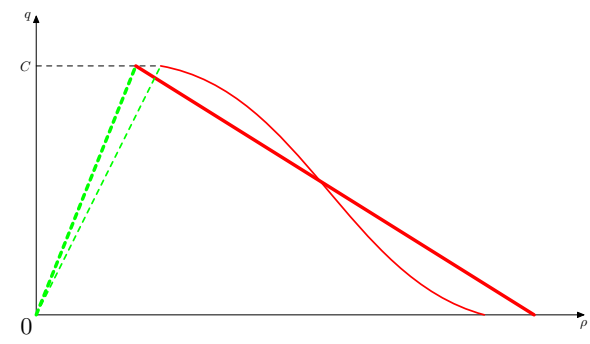

(a)

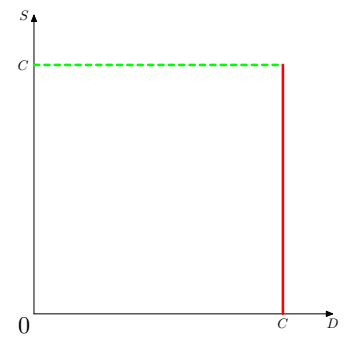

(b)

Fig. 1 The fundamental diagram and its supply-demand diagram

needed to compute the density $\rho$ from $(D, S)$. That is, $\rho$ can be written as a function of $(D, S)$ as

$$
\rho(D, S)= \begin{cases}D^{-1}(D), & D \leq S \\ S^{-1}(S), & S<D\end{cases}
$$

since $D(\rho)$ and $S(\rho)$ are invertible when the traffic is UC and OC respectively. Note that $\rho$ is not a function of $q$. If we introduce the supply-demand ratio $\gamma=D / S$, then $q(D, S)=\min \{\gamma, 1 / \gamma\} \cdot C$, and

$$
\rho(D, S)=R(\gamma) \equiv \begin{cases}D^{-1}(C \gamma), & \gamma \leq 1, \\ S^{-1}\left(\frac{C}{\gamma}\right), & \gamma>1,\end{cases}
$$

where $R(\gamma)$ is an increasing function in $\gamma \in[0, \infty]$. Here $R(0)=0, R(1)=\rho_{c}$, and $R(\infty)=\rho_{j}$. In this sense, $\rho=R(\gamma)$ can be considered as the inverse fluxdensity relationship. Similarly, $v=V(\rho)=V(R(\gamma))$ is a non-increasing function in $\gamma, V(0)=v_{f}$, and $V(\infty)=0$.

\subsection{The structure of solutions to the Riemann problem}

In supply-demand space, initial conditions (5] are equivalent to

$$
U(x, t=0)=\left\{\begin{array}{l}
U_{1}=\left(D_{1}, S_{1}\right), x<0 \\
U_{2}=\left(D_{2}, S_{2}\right), x>0 .
\end{array}\right.
$$

The Riemann problem at a linear boundary is then equivalent to the Riemann problem for (3) with initial conditions (23).

Unlike existing studies of hyperbolic conservation laws with discontinuous flux functions, in which solutions to the Riemann problem have been constructed in $\rho-q$ space, within the framework of wave separation developed in (Holden and Risebro 
1995), we construct the solutions to the Riemann problem for (3) with initial conditions (23) in supply-demand space.

In solutions to the Riemann problem for (3) with initial conditions (23), a shock wave or a rarefaction wave could initiate on a link from the linear boundary $x=0$, and traffic states on both links become asymptotically stationary after a long time. We denote the stationary state on the upstream link 1 by $U_{1}^{-}$and the stationary state on the downstream link 2 by $U_{2}^{+}$. At the boundary, there can also exist interior states (van Leer 1984; Bultelle et al. 1998), which take infinitesimal space. We denote the interior states on links 1 and 2 by $U_{1}\left(0^{-}, t\right)$ and $U_{2}\left(0^{+}, t\right)$ respectively. The structure of Riemann solutions on upstream and downstream links are shown in Figure 2

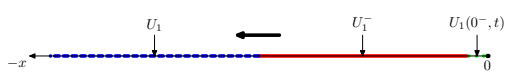

(a)

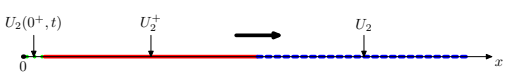

(b)

Fig. 2 Structure of Riemann solutions: (a) Upstream link 1; (b) Downstream link 2

Then the kinematic wave on the upstream link 1 is the solution of the corresponding homogeneous LWR model with initial left and right conditions of $U_{1}$ and $U_{1}^{-}$, respectively. Similarly, the kinematic wave on the downstream link 2 is the solution of the corresponding LWR model with initial left and right conditions of $U_{2}^{+}$and $U_{2}$, respectively. Since the stationary and interior states are constant for $t>0$, states on both links 1 and 2 are self-similar (Smoller 1983). That is, if stationary states exist and are unique, we have unique self-similar solutions for the Riemann problem of (3). In the following sections, we first derive necessary conditions for both stationary and interior states and then solve them in supply-demand space.

\section{Necessary conditions for the existence of stationary and interior states}

We denote $q_{1 \rightarrow 2}(0, t)$ as the flux from link 1 to link 2 . We first observe that the fluxes are determined by the stationary states: the asymptotic out-flux of link 1 is $q_{1}\left(0^{-}, t\right)=q\left(U_{1}^{-}\right)$, and the asymptotic in-flux of link 2 is $q_{2}\left(0^{+}, t\right)=q\left(U_{2}^{+}\right)$. Furthermore, from the conservation of traffic at the linear boundary, we have

$$
q_{1 \rightarrow 2}(0, t)=q_{1}\left(0^{-}, t\right)=q_{2}\left(0^{+}, t\right)=q\left(U_{1}^{-}\right)=q\left(U_{2}^{+}\right) .
$$




\subsection{The admissible conditions for stationary states}

As observed in (Holden and Risebro 1995, Coclite et al. 2005), the speed of the kinematic wave on an upstream link cannot be positive, and that on a downstream link cannot be negative. We have the following theorem.

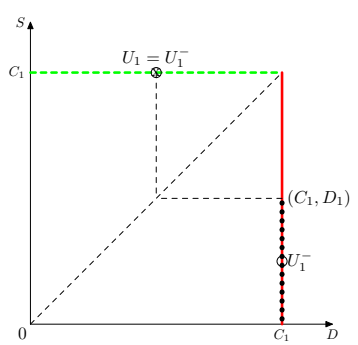

(a)

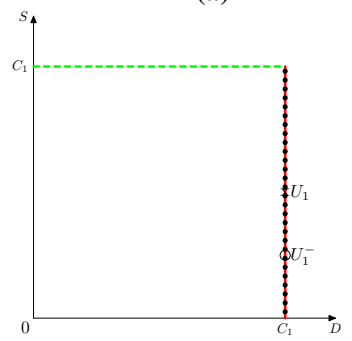

(c)

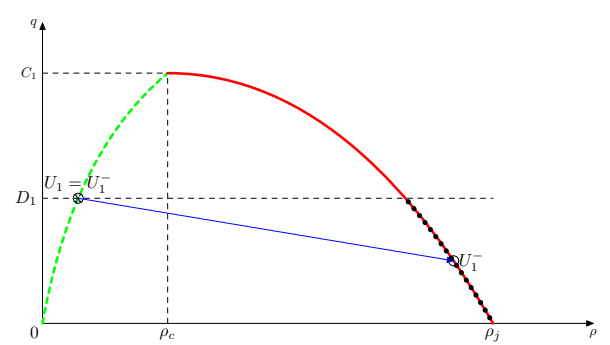

(b)

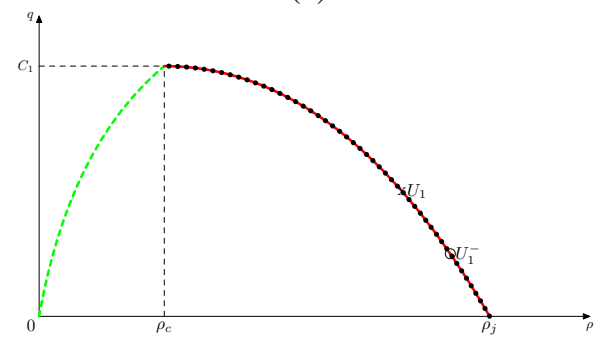

(d)

Fig. 3 Admissible stationary states for the upstream link 1

Theorem 1 (Admissible stationary states). For initial conditions in (23), stationary states are admissible if and only if

$$
U_{1}^{-}=\left(D_{1}, C_{1}\right) \text { or }\left(C_{1}, S_{1}^{-}\right)
$$

where $S_{1}^{-}<D_{1}$, and

$$
U_{2}^{+}=\left(C_{2}, S_{2}\right) \text { or }\left(D_{2}^{+}, C_{2}\right),
$$

where $D_{2}^{+}<S_{2}$. The results are illustrated in Figures 3 and 4

Proof. When the initial state on link 1 is strictly under-critical; i.e., when $D_{1}<S_{1}=$ $C_{1}$, the admissible stationary state $U_{1}^{-}$is the same as $U_{1}=\left(D_{1}, C_{1}\right)$ or strictly overcritical with $U_{1}^{-}=\left(C_{1}, S_{1}^{-}\right)$, where $S_{1}^{-}<D_{1}$. In this case, the Riemann problem for the LWR model on the upstream link 1 with upstream and downstream initial states $U_{1}=\left(D_{1}, C_{1}\right)$ and $U_{1}^{-}$has the following possible solutions: there is no wave when $U_{1}^{-}=U_{1}$; there is a backward traveling shock wave when $U_{1}^{-}=\left(C_{1}, S_{1}^{-}\right)$. In 


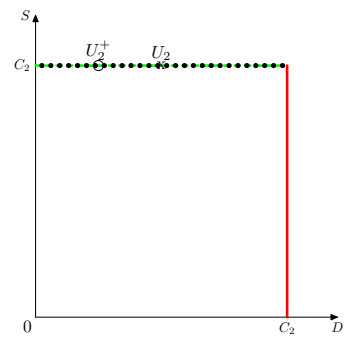

(a)

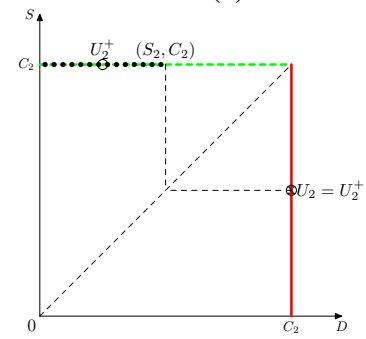

(c)

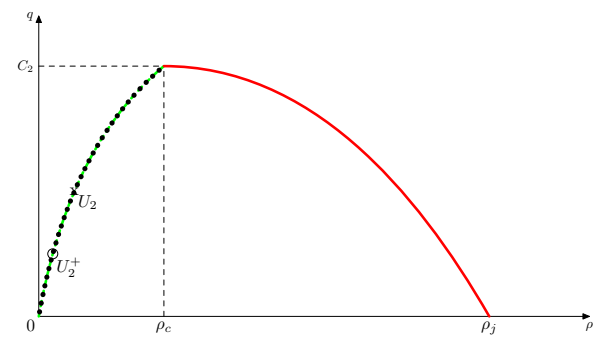

(b)

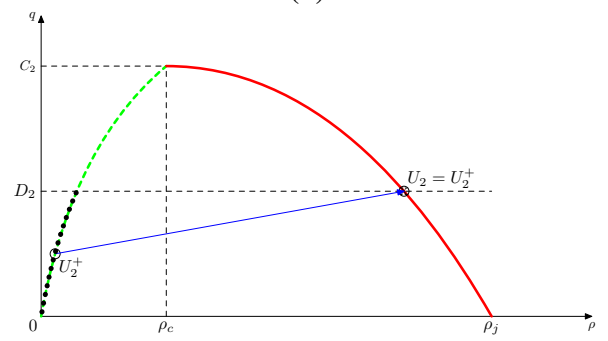

(d)

Fig. 4 Admissible stationary states for the downstream link 2

addition, we can verify that any stationary states not equal to $U_{1}$ and with $S_{1}^{-}>D_{1}$ will lead to forward traveling shock waves or rarefaction waves. Note that when $U_{1}^{-}=\left(C_{1}, D_{1}\right)$, the Riemann problem is solved by a zero shock wave, but $U_{1}^{-}$cannot be the stationary state by definition. When the initial state on link 1 is over-critical; i.e., when $S_{1} \leq D_{1}=C_{1}$, the admissible stationary state $U_{1}^{-}$is over-critical with $U_{1}^{-}=\left(C_{1}, S_{1}^{-}\right)$, where $S_{1}^{-} \leq C_{1}$. In this case, the Riemann problem for the LWR model on the upstream link 1 with upstream and downstream initial states $U_{1}=$ $\left(C_{1}, S_{1}\right)$ and $U_{1}^{-}=\left(C_{1}, S_{1}^{-}\right)$has the following possible solutions: there is no wave when $S_{1}^{-}=S_{1}$; there is a backward traveling shock wave when $S_{1}>S_{1}^{-}$; and there is a backward traveling rarefaction wave when $S_{1}<S_{1}^{-}$. Therefore, the stationary state is indeed admissible. In addition, we can verify that any strictly under-critical stationary states $U_{1}^{-}$will lead to forward traveling rarefaction waves and are not admissible.

When the initial state on link 2 is under-critical; i.e., when $D_{2} \leq S_{2}=C_{2}$, the admissible stationary state $U_{2}^{+}$is under-critical with $U_{2}^{+}=\left(D_{2}^{+}, C_{2}\right)$, where $D_{2}^{+} \leq$ $C_{2}$. In this case, the Riemann problem for the LWR model on the downstream link 2 with upstream and downstream initial states $U_{2}^{+}=\left(D_{2}^{+}, C_{2}\right)$ and $U_{2}=\left(D_{2}, C_{2}\right)$ has the following possible solutions: there is no wave when $D_{2}^{+}=D_{2}$; there is a forward traveling shock wave when $D_{2}^{+}<D_{2}$; there is a forward traveling rarefaction wave when $D_{2}^{+}>D_{2}$. Therefore, the stationary state is indeed admissible. In addition, we can verify that any strictly over-critical stationary states $U_{2}^{+}$will lead to backward traveling rarefaction waves and are not admissible. When the initial state on link 2 is strictly over-critical; i.e., when $S_{2}<D_{2}=C_{2}$, the admissible stationary state $U_{2}^{+}$is 
the same as $U_{2}=\left(C_{2}, S_{2}\right)$ or strictly under-critical with $U_{2}^{+}=\left(D_{2}^{+}, C_{2}\right)$, where $D_{2}^{+}<$ $S_{2}$. In this case, the Riemann problem for the LWR model on the downstream link 2 with upstream and downstream initial states $U_{2}^{+}$and $U_{2}=\left(D_{2}, C_{2}\right)$ has the following possible solutions: there is no wave when $U_{2}^{+}=U_{2}$; there is a forward traveling shock wave when $U_{2}^{+}=\left(D_{2}^{+}, C_{2}\right)$. In addition, we can verify that any stationary states not equal to $U_{2}$ and with $D_{2}^{+}>S_{2}$ will lead to backward traveling shock waves or rarefaction waves. Note that when $U_{2}^{+}=\left(S_{2}, C_{2}\right)$, the Riemann problem is solved by a zero shock wave, but $U_{2}^{+}$cannot be the stationary state by definition.

Remark 1 . Note that $U_{1}^{-}=U_{1}$ and $U_{2}^{+}=U_{2}$ are always admissible. In this case, the stationary states are the same as the corresponding initial states, and there are no waves.

Remark 2. From the proof of Theorem 1 , we can see that the types and traveling directions of waves on a homogeneous road can be solely determined by upstream demand and downstream supply and are not related to the shape of fundamental diagrams. This is why we are able to discuss the Riemann problem of the inhomogeneous LWR model in supply-demand space. Note that, however, the wave speeds are related to the details of the flux-density relation $Q(\rho)$.

Remark 3. Then the out-flux $q_{1}\left(0^{-}, t\right)=q\left(U_{1}^{-}\right)=\min \left\{D_{1}^{-}, S_{1}^{-}\right\} \leq D_{1}$ and the influx $q_{2}\left(0^{+}, t\right)=q\left(U_{2}^{+}\right)=\min \left\{D_{2}^{+}, S_{2}^{+}\right\} \leq S_{2}$. That is, $D_{1}$ is the maximum sending flux and $S_{2}$ is the maximum receiving flux in the sense of (Daganzo 1994, 1995a). Furthermore, $q_{1}\left(0^{-}, t\right)=D_{1}$, iff $U_{1}^{-}=\left(D_{1}, C_{1}\right)$, and iff $U_{1}^{-}$is UC; $q_{1}\left(0^{-}, t\right)<D_{1}$ iff $U_{1}^{-}=\left(C_{1}, S_{1}^{-}\right)$with $S_{1}^{-}<D_{1}$, and iff $U_{1}^{-}$is SOC. Similarly, $q_{2}\left(0^{+}, t\right)=S_{2}$, iff $U_{2}^{+}=\left(C_{2}, S_{2}\right)$, and iff $U_{2}^{+}$is OC; $q_{2}\left(0^{+}, t\right)<S_{2}$ iff $U_{2}^{+}=\left(D_{2}^{+}, C_{2}\right)$ with $D_{2}^{+}<S_{2}$, and iff $U_{2}^{+}$is SUC.

\subsection{The admissible conditions for interior states}

The Riemann problem on link 1 with left and right initial conditions of $U_{1}^{-}$and $U_{1}\left(0^{-}, t\right)$ cannot have negative waves. Otherwise, $U_{1}\left(0^{-}, t\right)$ will propagate upstream and violates the condition that it only exists at the boundary, but not anywhere upstream. Similarly, the Riemann problem on link 2 with left and right initial conditions of $U_{2}\left(0^{+}, t\right)$ and $U_{2}^{+}$cannot have positive waves. Therefore, interior states $U_{1}\left(0^{-}, t\right)$ and $U_{2}\left(0^{+}, t\right)$ should satisfy the following admissible conditions.

Theorem 2 (Admissible interior states). For asymptotic stationary states $U_{1}^{-}$and $U_{2}^{+}$, interior states $U_{1}\left(0^{-}, t\right)$ and $U_{2}\left(0^{+}, t\right)$ are admissible if and only if

$$
U_{1}\left(0^{-}, t\right)= \begin{cases}\left(C_{1}, S_{1}^{-}\right)=U_{1}^{-}, & \text {when } S_{1}^{-}<D_{1}^{-}=C_{1} \\ \left(D_{1}\left(0^{-}, t\right), S_{1}\left(0^{-}, t\right)\right), & \text { when } D_{1}^{-} \leq S_{1}^{-}=C_{1}\end{cases}
$$

where $S_{1}\left(0^{-}, t\right) \geq D_{1}^{-}$, and

$$
U_{2}\left(0^{+}, t\right)= \begin{cases}\left(D_{2}^{+}, C_{2}\right)=U_{2}^{+}, & \text {when } D_{2}^{+}<S_{2}^{+}=C_{2} \\ \left(D_{2}\left(0^{+}, t\right), S_{2}\left(0^{+}, t\right)\right), & \text { when } S_{2}^{+} \leq D_{2}^{+}=C_{2}\end{cases}
$$




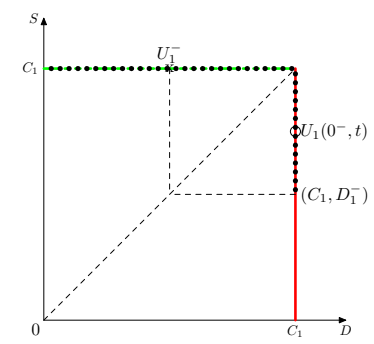

(a)

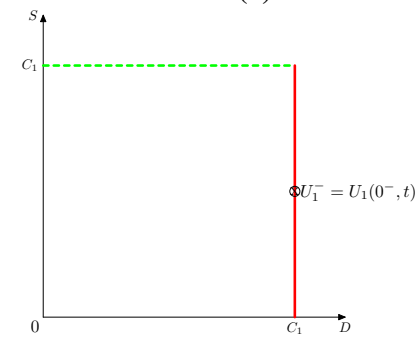

(c)

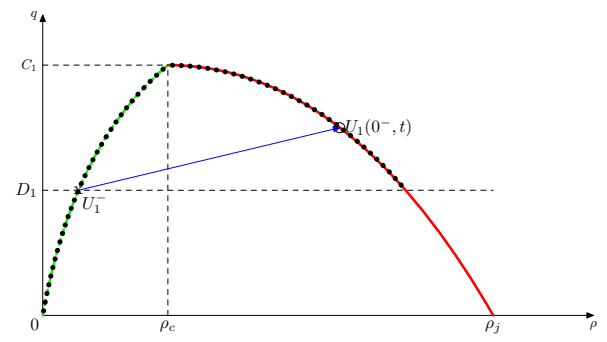

(b)

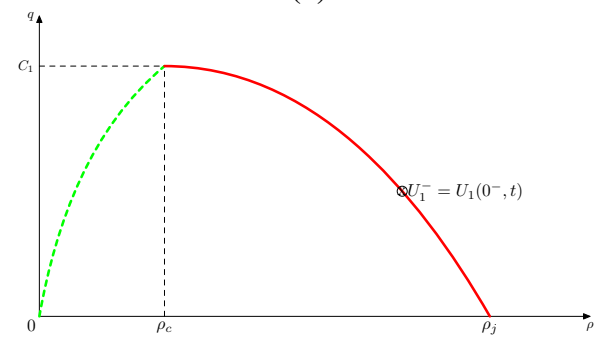

(d)

Fig. 5 Admissible interior states for upstream link 1

where $D_{2}\left(0^{+}, t\right) \geq S_{2}^{+}$. The results are illustrated in Figures 5 and 6 .

Proof. The results can be verified with the observation that the Riemann solutions for the homogeneous LWR model of the upstream links cannot have negative waves, and those of the downstream links cannot have positive waves.

Remark 1 . Note that $U_{1}\left(0^{-}, t\right)=U_{1}^{-}$and $U_{2}\left(0^{+}, t\right)=U_{2}^{+}$are always admissible. In this case, the interior states are the same as stationary states, and it is equivalent to saying that there are no interior states.

\subsection{An entropy condition for the local supply-demand method}

In addition to traffic conservation and admissible conditions, we introduce an entropy condition such that the boundary flux is always consistent with that by the supply-demand method (19) for the local interior states. At the boundary at $x=0$, the immediate upstream state is $U_{1}\left(0^{-}, t\right)$, and the immediate downstream state $U_{2}\left(0^{+}, t\right)$. That is, the interior states have to satisfy the following entropy condition

$$
q_{1 \rightarrow 2}(0, t)=\min \left\{D_{1}\left(0^{-}, t\right), S_{2}\left(0^{+}, t\right)\right\} .
$$

Note that the entropy condition (29) is also equivalent to the following localized optimization problem 


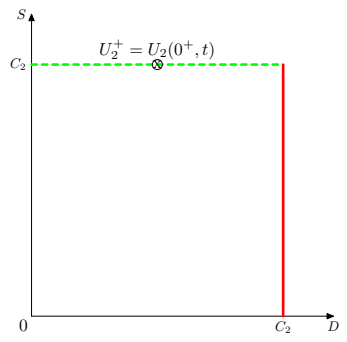

(a)

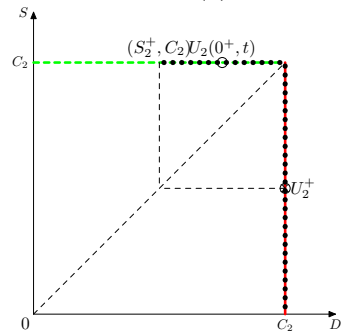

(c)

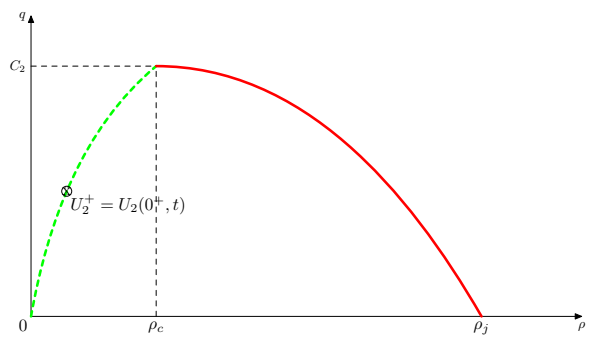

(b)

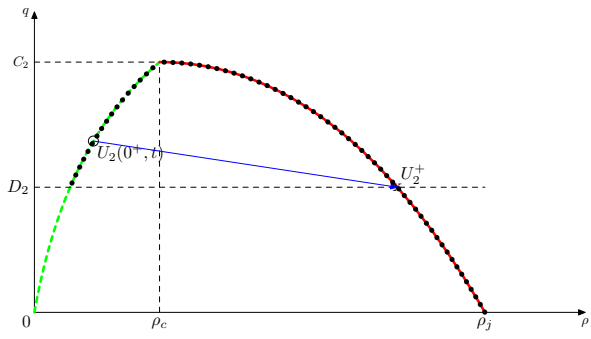

(d)

Fig. 6 Admissible interior states for downstream link 2

$$
\max \left\{q_{1 \rightarrow 2}(0, t)=q_{1}\left(0^{-}, t\right)=q_{2}\left(0^{+}, t\right)\right\}
$$

subject to

$$
\begin{aligned}
& q_{1}\left(0^{-}, t\right) \leq D_{1}\left(0^{-}, t\right), \\
& q_{2}\left(0^{+}, t\right) \leq S_{2}\left(0^{+}, t\right) .
\end{aligned}
$$

That is, the stationary and interior states are solutions of the optimization problem in the domain defined by the traffic conservation condition (24), the admissible conditions for stationary states $25 \mid 26$, and the admissible conditions for interior states $(27,28)$. Optimization formulations of entropy conditions were also adopted in (Holden and Risebro 1995; Coclite et al. 2005), but in terms of stationary states in flux-density space rather than in supply-demand space as we have done here.

In all of the necessary conditions above, we can see that the stationary and interior states are independent of the upstream supply, $S_{1}$, and the downstream demand, $D_{2}$. That is, the same upstream demand and downstream supply will yield the same solutions of stationary and interior states: when the upstream traffic is congested, its congestion level is not relevant to the stationary and interior states or the boundary flux; when the downstream traffic is free flow, its density is not relevant to the stationary and interior states or the boundary flux. Note that, however, the types and speeds of waves on both links can be related to $S_{1}$ as shown in Figure 3 (d) and $D_{2}$ as shown in Figure 4 (d). 


\section{Solutions to the Riemann problem for the inhomogeneous LWR model}

For the Riemann problem of (3) with initial conditions (23), we first solve for the stationary and interior states that satisfy the traffic conservation condition (24), the admissible conditions for stationary states $25 \mid 26$, the admissible conditions for interior states (27, 28), and the entropy condition (29). Then we compare them with existing solutions for both the homogeneous and inhomogeneous LWR models.

Lemma 1. In the Riemann solutions, the boundary flux satisfies

$$
q_{1 \rightarrow 2}(0, t)=q\left(U_{1}^{-}\right)=q\left(U_{2}^{+}\right)=\min \left\{D_{1}, S_{2}\right\} .
$$

Proof. From Theorem 1 and traffic conservation (24), we have that

$$
q_{1 \rightarrow 2}(0, t)=q\left(U_{1}^{-}\right)=q\left(U_{2}^{+}\right) \leq \min \left\{D_{1}, S_{2}\right\} .
$$

We demonstrate that it is not possible for $q_{1 \rightarrow 2}(0, t)<\min \left\{D_{1}, S_{2}\right\}$. Otherwise, we assume that $q_{1 \rightarrow 2}(0, t)=q_{0}<\min \left\{D_{1}, S_{2}\right\} \leq \min \left\{C_{1}, C_{2}\right\}$. Since $q\left(U_{1}^{-}\right)=q_{0}<D_{1}$, from 25 we have that $U_{1}^{-}=\left(C_{1}, q_{0}\right)$. Further from 27$)$ we have that $U_{1}\left(0^{-}, t\right)=$ $U_{1}^{-}=\left(C_{1}, q_{0}\right)$. Hence $D_{1}\left(0^{-}, t\right)=C_{1}$. Similarly, since $q\left(U_{2}^{+}\right)=q_{0}<S_{2}$, from 26) we have that $U_{2}^{+}=\left(q_{0}, C_{2}\right)$. Further from $\sqrt{28)}$ we have that $U_{2}\left(0^{+}, t\right)=U_{2}^{+}=$ $\left(q_{0}, C_{2}\right)$. Hence $S_{2}\left(0^{+}, t\right)=C_{2}$. Then from 29$)$ we have $q_{1 \rightarrow 2}(0, t)=\min \left\{C_{1}, C_{2}\right\}$, which contradicts the assumption that $q_{1 \rightarrow 2}(0, t)<\min \left\{C_{1}, C_{2}\right\}$. Therefore we have (30).

That is, the local optimal solution in 29) leads to a global optimal flux at a linear boundary, which satisfies the following optimization problem

$$
\max \left\{q_{1 \rightarrow 2}(0, t)\right\}
$$

subject to

$$
\begin{aligned}
& q_{1 \rightarrow 2}(0, t) \leq D_{1} \\
& q_{1 \rightarrow 2}(0, t) \leq S_{2}
\end{aligned}
$$

Therefore, in the Godunov finite difference equation (18), the boundary flux at the first time step by $(19)$ is the same as the asymptotic flux, regardless of the time step size. That is, the discrete flux in (19) is consistent with the continuous flux in 29.).

Theorem 3. The stationary states and interior states of the Riemann problem for (3) with initial conditions (23) are the following:

1. When $D_{1}<S_{2}$, we have unique stationary and interior states: $U_{1}^{-}=U_{1}\left(0^{-}, t\right)=$ $\left(D_{1}, C_{1}\right)$ and $U_{2}^{+}=U_{2}\left(0^{+}, t\right)=\left(D_{1}, C_{2}\right)$;

2. When $D_{1}>S_{2}$, we have unique stationary and interior states: $U_{1}^{-}=U_{1}\left(0^{-}, t\right)=$ $\left(C_{1}, S_{2}\right)$ and $U_{2}^{+}=U_{2}\left(0^{+}, t\right)=\left(C_{2}, S_{2}\right)$; 
3. When $D_{1}=S_{2}$, we have unique stationary states: $U_{1}^{-}=\left(D_{1}, C_{1}\right)$, and $U_{2}^{+}=$ $\left(C_{2}, S_{2}\right)$; but interior states may not be unique: $U_{1}\left(0^{-}, t\right)=\left(D_{1}, C_{1}\right)$ and $D_{2}\left(0^{+}, t\right) \geq$ $S_{2}$ and $S_{2}\left(0^{+}, t\right) \geq S_{2}$, or $U_{2}\left(0^{+}, t\right)=\left(C_{2}, S_{2}\right)$ and $S_{1}\left(0^{-}, t\right) \geq D_{1}$ and $D_{1}\left(0^{-}, t\right) \geq$ $D_{1}$.

Proof. When $D_{1}<S_{2} \leq C_{2}$, 30) leads to $q_{1 \rightarrow 2}(0, t)=D_{1}$. For link 1, since $q\left(U_{1}^{-}\right)=D_{1}$, from 25 we have that $U_{1}^{-}=\left(D_{1}, C_{1}\right)$. Further from 27] we have that $U_{1}\left(0^{-}, t\right)=\left(D_{1}\left(0^{-}, t\right), S_{1}\left(0^{-}, t\right)\right)$ with $S_{1}\left(0^{-}, t\right) \geq D_{1}^{-}=D_{1}$. For link 2 , since $q\left(U_{2}^{+}\right)=D_{1}<S_{2}$, from 26) we have that $U_{2}^{+}=\left(D_{1}, C_{2}\right)$. Further from 28) we have that $U_{2}\left(0^{+}, t\right)=U_{2}^{+}=\left(D_{1}, C_{2}\right)$. Then from 29) we have $q_{1 \rightarrow 2}(0, t)=$ $\min \left\{D_{1}\left(0^{-}, t\right), C_{2}\right\}=D_{1}$. Therefore, $D_{1}\left(0^{-}, t\right)=D_{1}$ and $S_{1}\left(0^{-}, t\right)=C_{1}$.

When $S_{2}<D_{1} \leq C_{1}$, 30 leads to $q_{1 \rightarrow 2}(0, t)=S_{2}$. For link 1, since $q\left(U_{1}^{-}\right)=$ $S_{2}<D_{1}$, from 25) we have that $U_{1}^{-}=\left(C_{1}, S_{2}\right)$. Further from 27) we have that $U_{1}\left(0^{-}, t\right)=U_{1}^{-}=\left(C_{1}, S_{2}\right)$. For link 2, since $q\left(U_{2}^{+}\right)=S_{2}$, from $\sqrt{26}$ we have that $U_{2}^{+}=\left(C_{2}, S_{2}\right)$. Further from 288 we have that $U_{2}\left(0^{+}, t\right)=\left(D_{2}\left(0^{+}, t\right), S_{2}\left(0^{+}, t\right)\right)$ with $D_{2}\left(0^{+}, t\right) \geq S_{2}$. Then from (29) we have $q_{1 \rightarrow 2}(0, t)=\min \left\{C_{1}, S_{2}\left(0^{+}, t\right)\right\}=S_{2}$. Therefore, $S_{2}\left(0^{+}, t\right)=S_{2}$ and $D_{2}\left(0^{+}, t\right)=C_{2}$.

When $D_{1}=S_{2}, 30$ leads to $q_{1 \rightarrow 2}(0, t)=D_{1}=S_{2}$. For link 1 , since $q\left(U_{1}^{-}\right)=D_{1}$, from 25 we have that $U_{1}^{-}=\left(D_{1}, C_{1}\right)$. Further from 27) we have that $U_{1}\left(0^{-}, t\right)=$ $\left(D_{1}\left(0^{-}, t\right), S_{1}\left(0^{-}, t\right)\right)$ with $S_{1}\left(0^{-}, t\right) \geq D_{1}^{-}=D_{1}$. For link 2 , since $q\left(U_{2}^{+}\right)=S_{2}$, from (26) we have that $U_{2}^{+}=\left(C_{2}, S_{2}\right)$. Further from 28) we have that $U_{2}\left(0^{+}, t\right)=$ $\left(D_{2}\left(0^{+}, t\right), S_{2}\left(0^{+}, t\right)\right)$ with $D_{2}\left(0^{+}, t\right) \geq S_{2}$. Then from (29) we have $q_{1 \rightarrow 2}(0, t)=$ $\min \left\{D_{1}\left(0^{-}, t\right), S_{2}\left(0^{+}, t\right)\right\}=D_{1}=S_{2}$. If $D_{1}\left(0^{-}, t\right)=D_{1}=S_{2}$, then $S_{1}\left(0^{-}, t\right)=C_{1}$, and $S_{2}\left(0^{+}, t\right) \geq S_{2}$. In this case, the interior state $U_{2}\left(0^{+}, t\right)$ may not be unique with $D_{2}\left(0^{+}, t\right) \geq S_{2}$ and $S_{2}\left(0^{+}, t\right) \geq S_{2}$. Note that, when $S_{2}=C_{2}$, the interior state is $U_{2}\left(0^{+}, t\right)=U_{2}^{+}$. If $S_{2}\left(0^{+}, t\right)=D_{1}=S_{2}$, then $D_{2}\left(0^{+}, t\right)=C_{2}$, and $D_{1}\left(0^{-}, t\right) \geq D_{1}$. In this case, the interior state $U_{1}\left(0^{-}, t\right)$ may not be unique with $S_{1}\left(0^{-}, t\right) \geq D_{1}$ and $D_{1}\left(0^{-}, t\right) \geq D_{1}$. Note that, when $D_{1}=C_{1}$, the interior state is $U_{1}\left(0^{-}, t\right)=U_{1}^{-}$. If both the upstream link 1 and the downstream link 2 have the same fundamental diagram, this case corresponds to a stationary shock, and the interior state is the same as that in (van Leer 1984).

Remark 1. From the theorem we can see that the stationary states always exist and are unique for the same pair of $D_{1}$ and $S_{2}$. Thus, with given $U_{1}$ and $U_{2}$, we can find unique kinematic waves on both links 1 and 2. Therefore, in the new solution framework, the solutions for the Riemann problem of the inhomogeneous LWR model always exist and are unique, although we may have multiple interior states.

Remark 2. If the entropy condition (29) is replaced by (30), we still have the same solutions $U_{1}^{-}$and $U_{2}^{+}$. That is, if we do not consider possible interior states as in (Seguin and Vovelle 2003; Garavello et al. 2007), then traffic conservation (24), admissible conditions for stationary states (25) 26), and the entropy condition (30) will yield the same stationary state solutions. However, this simplified approach - which is what currently exists in the literature - does not yield the existence or properties of the interior states.

Remark 3. When $D_{1}=S_{2}$, we have the following interior states that are different from the stationary states at $x=0^{-}$or $x=0^{+}$. The interior state at $x=0^{-}$has to 
satisfy $q\left(U_{1}\left(0^{-}, t\right)\right) \geq D_{1}$, and the interior state at $x=0^{+}$has to satisfy $q\left(U_{2}\left(0^{+}, t\right) \geq\right.$ $S_{2}$.

In addition, we have the following conclusions concerning possible stationary states at a linear boundary.

Corollary 1. When both links 1 and 2 reach asymptotic stationary states, they share the same flux $q$, and possible stationary states are the following: both links are UC with link 1 at $\left(q, C_{1}\right)$ and link 2 at $\left(q, C_{2}\right)$ where $q=D_{1}<S_{2}$; both links are OC with link 1 at $\left(C_{1}, q\right)$ and link 2 at $\left(C_{2}, q\right)$ where $q=S_{2}<D_{1}$; link 1 is UC at $\left(q, C_{1}\right)$ and link 2 OC at $\left(C_{2}, q\right)$ where $q=D_{1}=S_{2}$. It is not possible that link 1 is SOC and link 2 SUC.

Remark. The stationary states are stable in the sense that, when they are given as initial states, we obtain the same stationary states following Theorem 3

\subsection{The homogeneous LWR model}

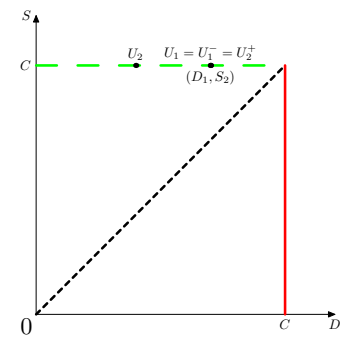

(a)

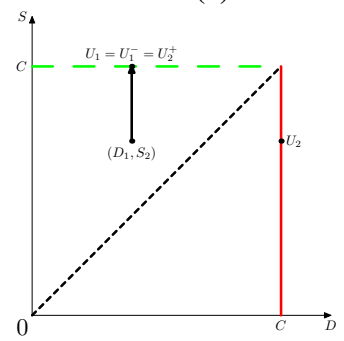

(d)

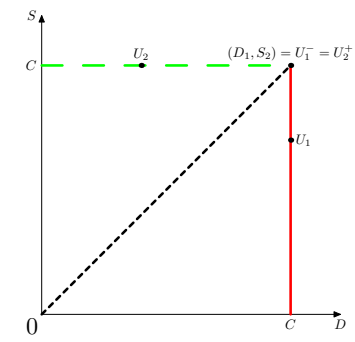

(b)

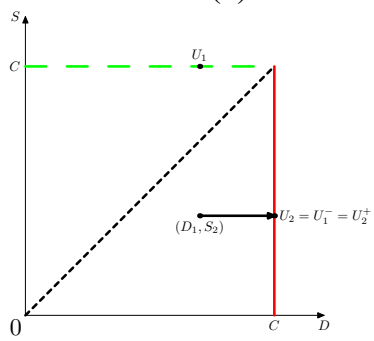

(e)

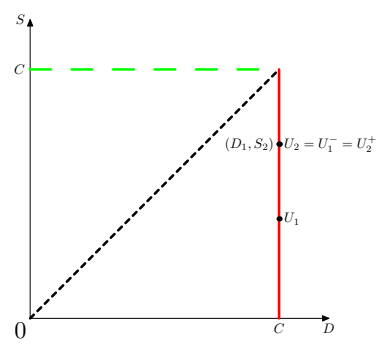

(c)

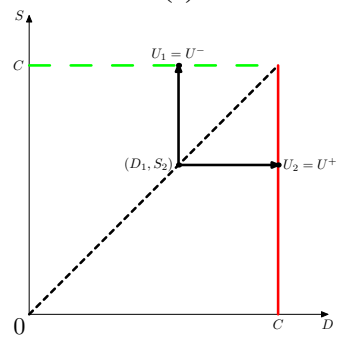

(f)

Fig. 7 The Riemann problem for the LWR model: Stationary states in supply-demand diagrams

For the original LWR model (2), the upstream link 1 and the downstream link 2 have the same fundamental diagram. Therefore we have $C_{1}=C_{2}$. In (Lebacque 1996), there are four scenarios for solutions to the Riemann problem. Here we reorganize them into the following six cases of initial conditions: 
Case 1 Link 1 is SUC, and link 2 is UC. That is, $D_{1}<S_{1}=C_{1}$ and $D_{2} \leq S_{2}=C_{2}$. In this case, $D_{1}<S_{2}$. From Theorem 3 we have that $U_{1}^{-}=U_{1}\left(0^{-}, t\right)=U_{2}^{+}=$ $U_{2}\left(0^{+}, t\right)=U_{1}$ and $q_{1 \rightarrow 2}(0, t)=q\left(U_{1}\right)$. Therefore, on link 1 , there is no wave; and on link 2, there is a forward shock wave when $D_{1}<D_{2}$, a forward rarefaction wave when $D_{1}>D_{2}$, and no wave when $D_{1}=D_{2}$.

Case 2 Link 1 is OC, and link 2 is UC. That is, $S_{1} \leq D_{1}=C_{1}$ and $D_{2} \leq S_{2}=C_{2}$. In this case, $D_{1}=S_{2}=C_{2}$. From Theorem 3 we have that $U_{1}^{-}=U_{1}\left(0^{-}, t\right)=U_{2}^{+}=$ $U_{2}\left(0^{+}, t\right)=\left(C_{1}, C_{2}\right)$ and $q_{1 \rightarrow 2}(0, t)=C_{1}$. Therefore, on link 1 , there is a backward rarefaction wave when $S_{1}<C_{1}$ and no wave when $S_{1}=C_{1}$; and on link 2, there is a forward rarefaction wave when $D_{2}<S_{2}$ and no wave when $D_{2}=S_{2}$.

Case 3 Link 1 is OC, and link 2 is SOC. That is, $S_{1} \leq D_{1}=C_{1}$ and $S_{2}<D_{2}=C_{2}$. In this case, $C_{1}=D_{1}>S_{2}$. From Theorem 3 we have that $U_{1}^{-}=U_{1}\left(0^{-}, t\right)=U_{2}^{+}=$ $U_{2}\left(0^{+}, t\right)=U_{2}$ and $q_{1 \rightarrow 2}(0, t)=q\left(U_{2}\right)$. Therefore, on link 1 there is a backward shock wave when $S_{1}>S_{2}$, a backward rarefaction wave when $S_{1}<S_{2}$, and no wave when $S_{1}=S_{2}$; and on link 2, there is no wave.

Case 4 Link 1 is SUC, and link 2 is OC, and $q\left(U_{1}\right)<q\left(U_{2}\right)$. That is, $D_{1}<S_{2} \leq D_{2}=$ $S_{1}=C_{1}$. From Theorem 3 we have that $U_{1}^{-}=U_{1}\left(0^{-}, t\right)=U_{2}^{+}=U_{2}\left(0^{+}, t\right)=U_{1}$ and $q_{1 \rightarrow 2}(0, t)=q\left(U_{1}\right)$. Therefore, on link 1 , there is no wave; and on link 2 , there is a forward shock wave.

Case 5 Link 1 is SUC, and link 2 is SOC, and $q\left(U_{1}\right)>q\left(U_{2}\right)$. That is, $S_{2}<D_{1} \leq S_{1}=$ $D_{2}=C_{1}$. From Theorem 3 we have that $U_{1}^{-}=U_{1}\left(0^{-}, t\right)=U_{2}^{+}=U_{2}\left(0^{+}, t\right)=U_{2}$ and $q_{1 \rightarrow 2}(0, t)=q\left(U_{2}\right)$. Therefore, on link 1 , there is a backward shock wave; and on link 2 , there is a no wave.

Case 6 Link 1 is SUC, and link 2 is SOC, and $q\left(U_{1}\right)=q\left(U_{2}\right)$. That is, $D_{1}=S_{2}<D_{2}=$ $S_{1}=C_{1}$. From Theorem 3 we have that $U_{1}^{-}=U_{2}^{+}=\left(D_{1}, S_{2}\right)$, and $q_{1 \rightarrow 2}(0, t)=$ $q\left(U_{1}\right)=q\left(U_{2}\right)$. Therefore, on link 1 , there is no wave; and on link 2 , there is no wave. In this case, there can exist interior states on link 1 or link 2: $U_{1}\left(0^{-}, t\right)=U_{1}^{-}$and $\min \left\{D_{2}\left(0^{+}, t\right), S_{2}\left(0^{+}, t\right)\right\} \geq D_{1}$ or $U_{2}\left(0^{+}, t\right)=U_{2}^{+}$and $\min \left\{D_{1}\left(0^{-}, t\right), S_{1}\left(0^{-}, t\right)\right\} \geq D_{1}$.

Obviously the results of stationary states and kinematic waves above are consistent with those in (Lebacque 1996). That is, the new solution framework yields the same wave solutions as traditional approaches.

The solutions of stationary states for the six cases are also shown in Figure 7 . where figures (a)-(f) are for cases 1-6 respectively. In these figures, both the upstream and downstream links share the same supply-demand diagram. From initial conditions $U_{1}$ and $U_{2}$ we can first draw the pair $\left(D_{1}, S_{2}\right)$, from which we can determine upstream and downstream stationary states accordingly. Further we can summarize the solutions of stationary states in Figure 8 in the $\left(D_{1}, S_{2}\right)$ space. This figure also demonstrates a graphical scheme for solving the stationary states as follows. First, from initial $U_{1}$ we draw a vertical line (thin pink line with an arrow), from initial $U_{2}$ we draw a horizontal line (thin pink line with an arrow), and the intersection point is $\left(D_{1}, S_{2}\right)$. Then, if the intersection point is above the line $0 A$, we draw a vertical line (thick blue line with arrow), and its intersection with $A C$ gives the stationary states; if the intersection point is below the line $0 A$, we draw a horizontal line (thick blue line with arrow), and its intersection with $A C$ gives the 
stationary states; if the intersection point is on the line $0 \mathrm{~A}$, we draw both a vertical line (thick blue line with arrow) and a horizontal line (thick blue line with arrow), and their intersections with $A C$ are the stationary states for the upstream and the downstream links respectively. Note that this scheme also works when the upstream and downstream links do not have the same fundamental diagrams but the same supply-demand diagram, i.e., $C_{1}=C_{2}$.

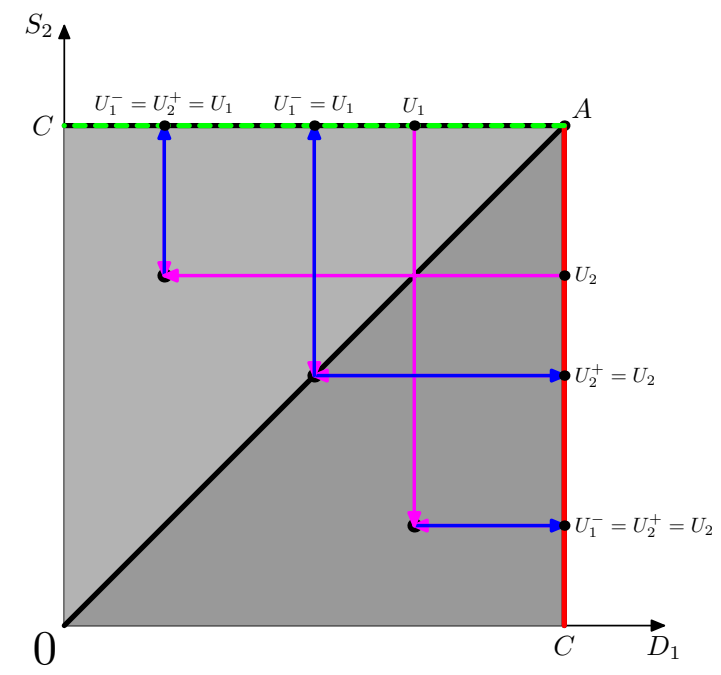

Fig. 8 Solution of stationary states for the Riemann problem for the LWR model

\subsection{The inhomogeneous LWR model}

When $C_{1} \neq C_{2}$, then the road is inhomogeneous, and there is a discontinuity in the fundamental diagram at $x=0$. In Figure 9 , we demonstrate a graphical scheme for solving stationary states in the $\left(D_{1}, S_{2}\right)$ space for the inhomogeneous LWR model. We take Figure 9 (a) as an example, in which $C_{1}<C_{2}$. First, from initial $U_{1}$ we draw a vertical line (thin pink line with an arrow), from initial $U_{2}$ we draw a horizontal line (thin pink line with an arrow), and the intersection point is $\left(D_{1}, S_{2}\right)$. Then, if the intersection point is above the line $0 A$, we draw a vertical line (thick blue line with arrow), and its intersections with $A C_{1}$ and $A C_{2}$ are the stationary states on links 1 and 2 respectively; if the intersection point $\left(D_{1}, S_{2}\right)$ is below the line $0 A$, we draw a horizontal line (thick blue line with arrow), and its intersections with $A C_{1}$ and $A C_{2}$ are the stationary states on links 1 and 2 respectively; if the intersection point $\left(D_{1}, S_{2}\right)$ is on the line $0 A$, we draw both a vertical line (thick blue line with arrow) and a horizontal line (thick blue line with arrow), and their intersections with 
$A C_{1}$ and $A C_{2}$ are the stationary states for the upstream and the downstream links respectively. This scheme is the same as that for the homogeneous LWR model.

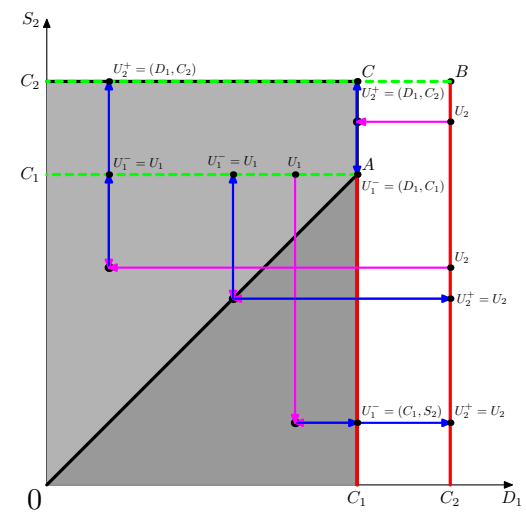

(a)

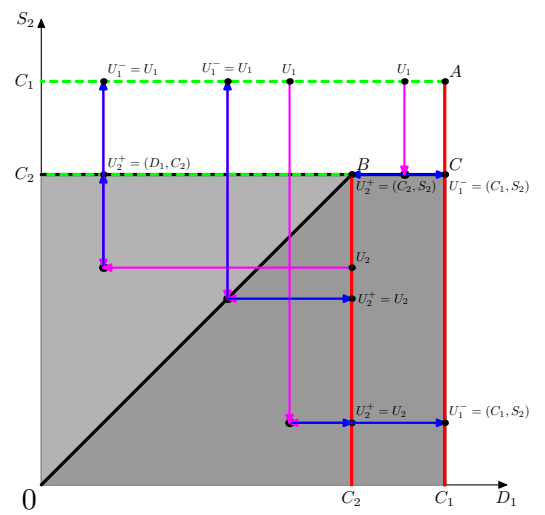

(b)

Fig. 9 Solution of stationary states of the Riemann problem for the inhomogeneous LWR model

In (Jin and Zhang 2003a), the Riemann problem for the inhomogeneous LWR model was solved as a resonant nonlinear system, and ten types of wave solutions were obtained. For example, wave solutions of Type 1 can be obtained in the new framework as follows. Both $U_{1}$ and $U_{2}$ are UC, $D_{2}<D_{1} \leq C_{2}=S_{2}$, and $C_{1}$ may be greater or smaller than $C_{2}$. From Figure 9 or Theorem 3 , we can see that $U_{1}^{-}=\left(D_{1}, C_{1}\right)=U_{1}, U_{2}^{+}=\left(D_{1}, C_{2}\right)$, there is no wave on link 1 , there is a forward rarefaction wave on link 2 , and $q_{1 \rightarrow 2}(0, t)=q\left(U_{1}\right)$. It is easy to check that the wave solutions of other types are also consistent.

\section{Asymptotic traffic dynamics on an inhomogeneous ring road}

In this section we consider the inhomogeneous ring road with length $L$ shown in Figure 10, in which the traffic direction is shown by the arrow. The ring road is composed of two homogeneous links: link 1 with capacity $C_{1}$ for $x \in\left[0, L_{1}\right]$, link 2 with capacity $C_{2}$ for $x \in\left[L_{1}, L\right]$, the upstream boundary of link 1 is denoted as boundary 1 , and the downstream boundary as boundary 2 . Here we assume that link 1 is a bottleneck; i.e., $C_{1}<C_{2}$. For example, such a bottleneck can be caused by a smaller number of lanes. We assume the fundamental relationships for two links as $q=Q_{1}(\rho)$ and $\rho=R_{1}(\gamma)$ for $x \in\left[0, L_{1}\right]$, and $q=Q_{2}(\rho)$ and $\rho=R_{2}(\gamma)$ for $x \in\left[L_{1}, L\right]$. 


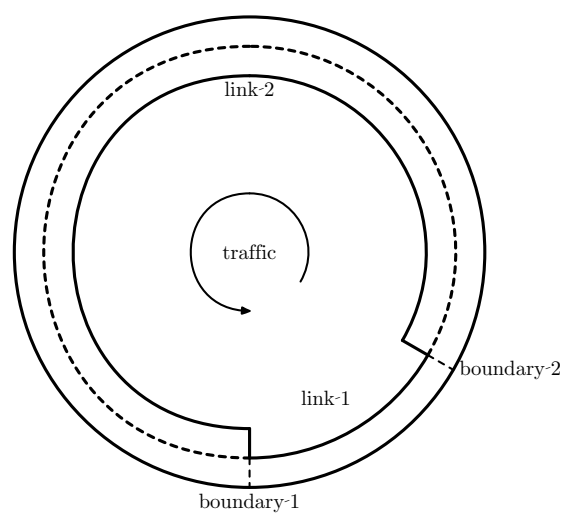

Fig. 10 A ring road

\subsection{Asymptotic stationary and interior states}

When the ring road reaches asymptotic stationary states, the flux at any location is the same, e.g., q. As we know, the asymptotic stationary state on a link can be uniformly UC, uniformly SOC, or a stationary shock wave (SS) connecting an upstream SUC state and a downstream SOC state (Bultelle et al. 1998). Then all possible combinations of stationary states are listed in Table 1 and explained in the following.

- When link 1 is UC at $\left(q, C_{1}\right)$ with $q \leq C_{1}$, we have the following scenarios. (a) From Theorem 3 it is possible that link 2 is UC at $\left(q, C_{2}\right)$, and the total number of vehicles on the ring road is $N_{a}=R_{1}\left(q / C_{1}\right) L_{1}+R_{2}\left(q / C_{2}\right)\left(L-L_{1}\right)$. (b) If link 2 is SS with upstream $\left(q, C_{2}\right)$ and downstream $\left(C_{2}, q\right)$, we have that $q=C_{1}$ and link 1 is critical at $\left(C_{1}, C_{1}\right)$. Assuming that link 2 is SUC for $x \in\left[L_{1}, L_{2}\right]$ and SOC for $x \in\left[L_{2}, L\right]$. In this case, the total number of vehicles on the ring road is $N_{b}=R_{1}(1) L_{1}+R_{2}\left(C_{1} / C_{2}\right)\left(L_{2}-L_{1}\right)+R_{2}\left(C_{2} / C_{1}\right)\left(L-L_{2}\right)$. (c) If link 2 is SOC at $\left(C_{2}, q\right)$, we have from Theorem 3 that $q=S_{2}=C_{1}$ at boundary 1 . That is, link 1 is critical at $\left(C_{1}, C_{1}\right)$. In this case, the total number of vehicles on the ring road is $N_{c}=R_{1}(1) L_{1}+R_{2}\left(C_{2} / C_{1}\right)\left(L-L_{1}\right)$.

- When link 1 is SOC at $\left(C_{1}, q\right)$ with $q<C_{1}$, we have the following scenarios. (d) It is possible that link 2 is SOC at $\left(C_{2}, q\right)$, and the total number of vehicles on the ring road is $N_{d}=R_{1}\left(C_{1} / q\right) L_{1}+R_{2}\left(C_{2} / q\right)\left(L-L_{1}\right)$. If link 2 is UC at $\left(q, C_{2}\right)$, we have from Theorem 3 that $q=C_{1}$ at boundary 2 . If link 2 is SS with upstream $\left(q, C_{2}\right)$, we have from Theorem 3 that $q=C_{1}$ at boundary 2 . Thus these two scenarios are impossible, since $q=C_{1}$ contradicts $q<C_{1}$.

- When link 1 is SS with upstream $\left(q, C_{1}\right)$ and downstream $\left(C_{1}, q\right)$ with $q<C_{1}$, we have the following scenarios. If link 2 is $\mathrm{UC}$ at $\left(q, C_{2}\right)$, we have from Theorem 3 that $q=\min \left\{C_{1}, C_{2}\right\}=C_{1}$ at boundary 2 ; if link 2 is SS with upstream $\left(q, C_{2}\right)$, we have from Theorem 3 that $q=\min \left\{C_{1}, C_{2}\right\}=C_{1}$ at boundary 2; if link 2 is 
SOC at $\left(C_{2}, q\right)$, we have from Theorem 3 that $q=\min \left\{C_{2}, C_{1}\right\}=C_{1}$ at boundary 1. All three of these scenarios are impossible, since $q=C_{1}$ contradicts $q<C_{1}$.

\begin{tabular}{|c|c|c|c|}
\hline Link 1 & $\mathrm{UC}\left(q, C_{2}\right)$ & $\mathrm{SS}\left(q, C_{2}\right) \rightarrow\left(C_{2}, q\right)$ & $\mathrm{SOC}\left(C_{2}, q\right)$ \\
\hline $\mathrm{UC}\left(q, C_{1}\right)$ & (a) & (b) & (c) \\
\hline $\mathrm{SOC}\left(C_{1}, q\right)$ & $\mathrm{x}$ & $\mathrm{x}$ & (d) \\
\hline $\mathrm{SS}\left(q, C_{1}\right) \rightarrow\left(C_{1}, q\right)$ & $\mathrm{x}$ & $\mathrm{x}$ & $\mathrm{x}$ \\
\hline
\end{tabular}

Table 1 All possible stationary states on the ring road in Figure 10

For all four scenarios of asymptotic stationary states on the ring road, different scenarios have a different number of vehicles since

$$
\begin{array}{r}
0 \leq N_{a} \leq R_{1}(1) L_{1}+R_{2}\left(C_{1} / C_{2}\right)\left(L-L_{1}\right)<N_{b}<N_{c}= \\
r_{i}(1) L_{1}+R_{2}\left(C_{2} / C_{1}\right)\left(L-L_{1}\right)<N_{d} \leq R_{1}(\infty) L_{1}+R_{2}(\infty)\left(L-L_{1}\right) .
\end{array}
$$

Due to traffic conservation on the ring road, we can therefore determine the final stationary states by the initial number of vehicles $N$ on the road as follows: (a) When $N \leq R_{1}(1) L_{1}+R_{2}\left(C_{1} / C_{2}\right)\left(L-L_{1}\right)$, links 1 and 2 will be asymptotically stationary at UC with $\left(q, C_{1}\right)$ and $\left(q, C_{2}\right)$ respectively, where $q$ is the solution of $R_{1}\left(q / C_{1}\right) L_{1}+R_{2}\left(q / C_{2}\right)\left(L-L_{1}\right)=N$; (b) When $R_{1}(1) L_{1}+R_{2}\left(C_{1} / C_{2}\right)\left(L-L_{1}\right)<$ $N<R_{1}(1) L_{1}+R_{2}\left(C_{2} / C_{1}\right)\left(L-L_{1}\right)$, link 1 will be asymptotically stationary at critical with $\left(C_{1}, C_{1}\right)$, and link 2 at SS with $\left(C_{1}, C_{2}\right)$ for $x \in\left[L_{1}, L_{2}\right]$ and $\left(C_{2}, C_{1}\right)$ for $x \in$ $\left(L_{2}, L\right]$, where $L_{2}$ is the solution of $R_{1}(1) L_{1}+R_{2}\left(C_{1} / C_{2}\right)\left(L_{2}-L_{1}\right)+R_{2}\left(C_{2} / C_{1}\right)(L-$ $\left.L_{2}\right)=N$; (c) When $N=R_{1}(1) L_{1}+R_{2}\left(C_{2} / C_{1}\right)\left(L-L_{1}\right)$, link 1 will be asymptotically stationary at critical with $\left(C_{1}, C_{1}\right)$, and link 2 at SOC with $\left(C_{2}, C_{1}\right)$; (d) When $N>R_{1}(1) L_{1}+R_{2}\left(C_{2} / C_{1}\right)\left(L-L_{1}\right)$, links 1 and 2 will be asymptotically stationary at SOC with $\left(C_{1}, q\right)$ and $\left(C_{2}, q\right)$ respectively, where $q$ is the solution of $R_{1}\left(C_{1} / q\right) L_{1}+R_{2}\left(C_{2} / q\right)\left(L-L_{1}\right)=N$.

From Theorem 3 an interior state can occur at a boundary when its upstream demand equals the downstream supply, and its flux cannot be smaller than the demand or supply. In the following we consider possible asymptotic interior states on the ring road in Figure 10. First, at any location inside a uniform traffic stream on a homogeneous road, it is not possible to have interior states, since the upstream and downstream states are exactly the same at $(D, S)$ and $D=S$ if and only if the traffic is $D=S=C$, in which case the interior states have to be the same as the stationary states. Thus, interior states can only exist around the interface between two uniform traffic streams when the upstream demand equals the downstream supply, and we examine possible interior states in all four scenarios as follows. (a) The necessary condition for an interior state to exist at boundary 1 is $q=C_{1}$, i.e., when $N=R_{1}(1) L_{1}+R_{2}\left(C_{1} / C_{2}\right)\left(L-L_{1}\right)$. From Theorem 3 the interior state can only exist at $x=0^{-}$, but not $x=0^{+}$. The necessary condition for an interior state to exist at boundary 2 is $q=C_{2}$, which is not possible. (b) It is not possible for interior 
states to exist at either boundary 1 or 2, but it is possible for an interior state to exist around the SS interface at $x=L_{2}$. From Theorem 3 , the interior state can only exist at $x=L_{2}^{-}$or $x=L_{2}^{+}$. (c) It is not possible for an interior state to exist at boundary 1 , but it is possible for an interior state to exist around boundary 2 . That is, when $N=R_{1}(1) L_{1}+R_{2}\left(C_{1} / C_{2}\right)\left(L-L_{1}\right)$, from Theorem 3 , the interior state can only exist at $x=L_{1}^{+}$, but not $x=L_{1}^{-}$. (d) It is not possible for interior states to exist at either boundary 1 or 2 .

In summary, there can exist three types of interior states: (a) When $N=N_{1}=$ $R_{1}(1) L_{1}+R_{2}\left(C_{1} / C_{2}\right)\left(L-L_{1}\right)$, an interior state can only exist at $x=0^{-}=L^{-}$; (b) When $R_{1}(1) L_{1}+R_{2}\left(C_{1} / C_{2}\right)\left(L-L_{1}\right)<N<R_{1}(1) L_{1}+R_{2}\left(C_{2} / C_{1}\right)\left(L-L_{1}\right)$, an interior state can exist at $x=L_{2}^{-}$or $x=L_{2}^{+}$; (c) When $N=N_{3}=R_{1}(1) L_{1}+$ $R_{2}\left(C_{2} / C_{1}\right)\left(L-L_{1}\right)$, an interior state can only exist at $x=L_{1}^{+}$.

\subsection{Numerical examples}

In this subsection, we study asymptotic traffic dynamics on the inhomogeneous ring road in Figure 10 with $L=600 \mathrm{l}=16.8 \mathrm{~km}, L_{1}=100 \mathrm{l}=2.8 \mathrm{~km}$, and the locationdependent speed-density relationships are based on (Kerner and Konhäuser 1994. Herrmann and Kerner 1998)

$$
V(\rho, a(x))=5.0461\left[\left(1+\exp \left\{\left[\frac{\rho}{a(x) \rho_{j}}-0.25\right] / 0.06\right\}\right)^{-1}-3.72 \times 10^{-6}\right] l / \tau,
$$

where the relaxation time $\tau=5 \mathrm{~s}$; the unit length $l=0.028 \mathrm{~km}$; the free flow speed $v_{f}=27.8 \mathrm{~m} / \mathrm{s}$; the jam density of a single lane $\rho_{j}=180 \mathrm{veh} / \mathrm{km} / \mathrm{lane}$. Here the number of lanes $a(x)=1$ for link 1 and $a(x)=2$ for link 2 . The corresponding fundamental diagram $q=Q(\rho, a(x))$ is non-convex but unimodal in density $\rho$. In addition, $C_{1}=0.7091 \mathrm{veh} / \mathrm{s}$, and $C_{2}=2 C_{1}$. Thus we can compute $R_{1}(1)=35.8944$ $\mathrm{veh} / \mathrm{km}, R_{2}\left(C_{1} / C_{2}\right)=R_{2}\left(\frac{1}{2}\right)=26.4162 \mathrm{veh} / \mathrm{km}$, and $R_{2}\left(C_{2} / C_{1}\right)=R_{2}(2)=118.3550$ $\mathrm{veh} / \mathrm{km}$. Hence $N_{1}=R_{1}(1) L_{1}+R_{2}\left(C_{1} / C_{2}\right)\left(L-L_{1}\right)=470.3311 \mathrm{veh}$, and $N_{3}=$ $R_{1}(1) L_{1}+R_{2}\left(C_{2} / C_{1}\right)\left(L-L_{1}\right)=1757.4746$ veh.

Here we consider the following initial condition:

$$
\begin{array}{ll}
\rho(x, 0)=a(x)\left(\rho_{0}+3 \sin \frac{2 \pi x}{L}\right), & x \in[0, L], \\
v(x, 0)=V(\rho(x, 0), a(x)), & x \in[0, L] .
\end{array}
$$

Then, the total number of vehicles on the ring road is

$$
N=2 \rho_{0} L-\int_{0}^{100 l}\left(\rho_{0}+3 \sin \frac{2 \pi x}{L}\right) d x=1100 l \rho_{0}-\frac{450}{\pi} l
$$

When $\rho_{0}=15.4007 \mathrm{veh} / \mathrm{km}, N=N_{1}$ and we observe an interior state at $x=0^{-}=$ $L^{-}$; when $\rho_{0}=57.1911 \mathrm{veh} / \mathrm{km}, N=N_{3}$ and we observe an interior state at $x=L_{1}^{+}$; when $\rho_{0} \in(15.4007,57.1911)$, we observe an interior state at $x=L_{2}^{-}$or $x=L_{2}^{+}$, 
where $L_{2}$ is the solution of $R_{1}(1) L_{1}+R_{2}\left(\frac{1}{2}\right)\left(L_{2}-L_{1}\right)+R_{2}(2)\left(L-L_{2}\right)=N$. For example, when $\rho_{0}=28 \mathrm{veh} / \mathrm{km}, N=858.3893 \mathrm{veh}$, and

$$
L_{2}=\frac{N-\left(R_{1}(1)-R_{2}\left(\frac{1}{2}\right)\right) L_{1}-R_{2}(2) L}{R_{2}\left(\frac{1}{2}\right)-R_{2}(2)}=449.2561 l .
$$
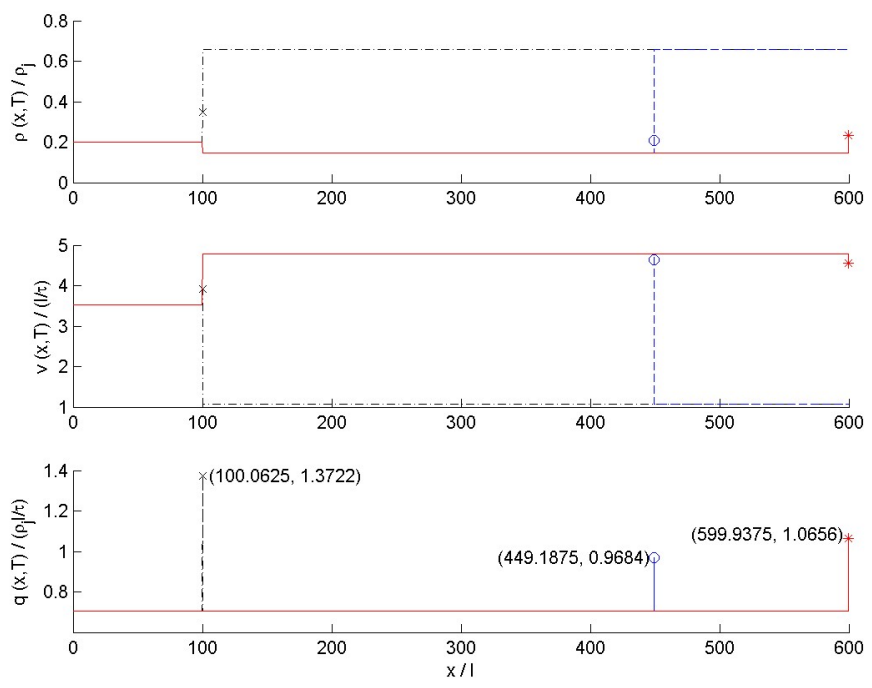

Fig. 11 Solutions of $\rho, v$, and $q$ at $T=24000 \mathrm{~s}$ for initial conditions in 31 : solid lines with stars for $\rho_{0}=15.4007 \mathrm{veh} / \mathrm{km}$, dashed lines with circles for $\rho_{0}=28 \mathrm{veh} / \mathrm{km}$, and dash-dotted lines for $\rho_{0}=57.1911$.

In the following, we simulate traffic dynamics on the ring road for three different initial $\rho_{0}: 15.4007 \mathrm{veh} / \mathrm{km}, 28 \mathrm{veh} / \mathrm{km}$, and $57.1911 \mathrm{veh} / \mathrm{km}$. Here we use the Godunov finite difference equation in (18) and the supply-demand method in (19) for computing boundary fluxes. The simulation time is $T=4800 \tau=24000 \mathrm{~s}$. We partition the road $[0, L]$ into $N=4800$ cells and the time interval $[0, T]$ into $K=240000$ steps. Hence, the length of each cell is $\Delta x=3.5 \mathrm{~m}$ and the length of each time step is $\Delta t=0.1 \mathrm{~s}$. The CFL condition number (Courant et al. 1928) is $v_{f} \frac{\Delta t}{\Delta x} \leq 0.79<1$. The results for the three initial conditions are shown in Figure 11, where the bottom figure shows the locations and fluxes of all three interior states. From the figure, we can see that each of the three interior states only exists in one cell, and the locations of interior states are exactly as predicted above. Note that the top right figure of Figure 18 in (Jin and Zhang 2003a) also demonstrates the existence of an interior state, which is at the interface of a stationary shock. 


\section{Conclusion}

In this paper we first reviewed the definitions of the supply and demand functions and the discrete supply-demand method for computing boundary fluxes. We then introduced the supply-demand diagram of a roadway and a new framework for solving the Riemann problem of the inhomogeneous LWR model in supply-demand space. In this framework, each link can have asymptotic interior and stationary states near the boundary, and the wave on each link is determined by the Riemann problem of the homogeneous LWR model with stationary and initial states for initial conditions. We have derived conditions for admissible stationary and interior states and introduced an entropy condition based on the discrete supply-demand method for computing boundary fluxes. We then proved that solutions to the Riemann problem exist and are unique and demonstrated that these solutions are consistent with those in literature for both the homogeneous and inhomogeneous LWR models. We also presented a graphical approach for finding the asymptotic stationary states with the help of supply-demand diagrams. Finally, we discussed the asymptotic stationary states on a ring road with arbitrary initial conditions and demonstrated with numerical examples that the existence and properties of the interior states are as predicted in this framework.

Unlike existing studies of the homogeneous or inhomogeneous LWR models, this study analyzes traffic dynamics in supply-demand space. In this framework, the discrete supply-demand method is applied as an entropy condition. In this sense, our study provides a new approach for constructing convergent solutions of finite difference equations arising in a Godunov method (18) with a supply-demand method 19] for both the homogeneous and inhomogeneous LWR models. We have demonstrated that this new approach can successfully predict the existence and properties of interior states in numerical solutions. However, note that interior states take only one cell in numerical solutions and vanish as we diminish the cell size. In this sense, the interior states are inconsequential to solutions of the Riemann problem.

Compared with existing studies, the new approach in the supply-demand framework is much simpler. In addition, since supply-demand methods have been proposed for computing fluxes through other junctions in general road networks (Daganzo 1995a; Lebacque 1996; Jin and Zhang 2003b; Jin|2003), our framework could be extended to constructing solutions to the Riemann problem in these models. In (Jin 2009), we successfully applied this framework to analyze the Riemann problem of merging traffic flow. In addition, one could also apply this new framework to analyze asymptotic traffic dynamics in a road network, such as the diverge-merge network studied in (Jin 2008).

Acknowledgements The work of the first two authors was supported in part by the National Natural Science Foundation of China (No. 50708107), the Hi-Tech Research and Development Program of China (863 Project) (No. 2007AA11Z222), and the National Basic Research Program of China (973 Project) (No. 2006CB705506). The work of the third author was supported in part by the US Department of Energy (DOE) Mathematical, Information, and Computing Sciences Division under contract number DE-FG02-03ER25579. 


\section{References}

Bale, D., LeVeque, R., Mitran, S., and Rossmanith, J. (2002). A Wave Propagation Method for Conservation Laws and Balance Laws with Spatially Varying Flux Functions. SIAM JOURNAL ON SCIENTIFIC COMPUTING, 24(3):955-978.

Bui, D., Nelson, P., and Narasimhan, S. (1992). Computational Realizations of the Entropy Condition in Modeling Congested Traffic Flow. Technical report.

Bultelle, M., Grassin, M., and Serre, D. (1998). Unstable Godunov discrete profiles for steady shock waves. SIAM J. Numer. Anal, 35(6):2272-2297.

Burger, R., Garcia, A., Karlsen, K., Towers, J., Tosin, A., Ambrosio, L., Crippa, G., LeFloch, P., Donato, P., Gaveau, F., et al. (2008). Difference schemes, entropy solutions, and speedup impulse for an inhomogeneous kinematic traffic flow model. NETWORKS AND HETEROGENEOUS MEDIA, 3(1):1.

Burger, R., Karlsen, K., Mishra, S., and Towers, J. (2005). On conservation laws with discontinuous flux. Trends in Applications of Mathematics to Mechanics, Shaker Verlag, Aachen, pages 75-84.

Coclite, G., Garavello, M., and Piccoli, B. (2005). Traffic flow on a road network. SIAM journal on mathematical analysis, 36(6):1862-1886.

Colella, P. and Puckett, E. G. (2004). Modern Numerical Methods for Fluid Flow. In draft.

Courant, R., Friedrichs, K., and Lewy, H. (1928). ber die partiellen differenzengleichungen der mathematischen physik. Mathematische Annalen, 100:32-74.

Daganzo, C. (2006). On the variational theory of traffic flow: well-posedness, duality and applications. NETWORKS AND HETEROGENEOUS MEDIA, 1(4):601.

Daganzo, C. F. (1994). The cell transmission model: a dynamic representation of highway traffic consistent with hydrodynamic theory. Transportation Research B, 28(4):269-287.

Daganzo, C. F. (1995a). The cell transmission model II: Network traffic. Transportation Research $B, 29(2): 79-93$.

Daganzo, C. F. (1995b). A finite difference approximation of the kinematic wave model of traffic flow. Transportation Research B, 29(4):261-276.

Daganzo, C. F. (1997). A continuum theory of traffic dynamics for freeways with special lanes. Transportation Research B, 31(2):83-102.

Diehl, S. (1995). On scalar conservation laws with point source and discontinuous flux function. SIAM Journal on Mathematical Analysis, 26(6):1425-1451.

Diehl, S. (1996a). A Conservation Law with Point Source and Discontinuous Flux Function Modelling Continuous Sedimentation. SIAM JOURNAL ON APPLIED MATHEMATICS, 56:388419.

Diehl, S. (1996b). Scalar conservation laws with discontinuous flux function: I. The viscous profile condition. Communications in Mathematical Physics, 176(1):23-44.

Diehl, S. and Wallin, N. (1996). Scalar conservation laws with discontinuous flux function: II. On the stability of the viscous profiles. Communications in Mathematical Physics, 176(1):45-71.

Engquist, B. and Osher, S. (1980a). One-Sided Difference Schemes and Transonic Flow. Proceedings of the National Academy of Sciences, 77(6):3071-3074.

Engquist, B. and Osher, S. (1980b). Stable and entropy satisfying approximations for transonic flow calculations. 34(149):45-75.

Engquist, B. and Osher, S. (1981). One-sided difference approximations for nonlinear conservation laws. Mathematics of Computation, 36(154):321-351.

Garavello, M., Natalini, R., Piccoli, B., and Terracina, A. (2007). Conservation laws with discontinuous flux. Netw. Heterog. Media, 2:159-179.

Gazis, D. C., Herman, R., and Rothery, R. W. (1961). Nonlinear follow-the-leader models of traffic flow. Operations Research, 9(4):545-567.

Gimse, T. (1993). Conservation Laws with Discontinuous Flux Functions. SIAM Journal on Mathematical Analysis, 24:279.

Gimse, T. and Risebro, N. (1990). Riemann problems with a discontinuous flux function. In Hyperbolic problems. Theory, numerical methods and applications. Vol. I, Proc. Conf., Uppsala/Sweden.

Godunov, S. K. (1959). A difference method for numerical calculations of discontinuous solutions of the equations of hydrodynamics. Matematicheskii Sbornik, 47:271-306. In Russian.

Greenshields, B. D. (1935). A study in highway capacity. Highway Research Board Proceedings, $14: 448-477$.

Herrmann, M. and Kerner, B. S. (1998). Local cluster effect in different traffic flow models. Physica A, 255:163-198.

Herty, M., Seaïd, M., and Singh, A. (2007). A domain decomposition method for conservation laws with discontinuous flux function. Applied Numerical Mathematics, 57(4):361-373. 
Holden, H. and Risebro, N. H. (1995). A mathematical model of traffic flow on a network of unidirectional roads. SIAM Journal on Mathematical Analysis, 26(4):999-1017.

Isaacson, E. I. and Temple, J. B. (1992). Nonlinear resonance in systems of conservation laws. SIAM Journal on Applied Mathematics, 52(5):1260-1278.

Jin, W.-L. (2003). Kinematic Wave Models of Network Vehicular Traffic. $\mathrm{PhD}$ thesis, University of California, Davis. http://arxiv.org/abs/math.DS/0309060.

Jin, W.-L. (2008). Asymptotic traffic dynamics arising in diverge-merge networks with two intermediate links. Transportation Research Part B. In press.

Jin, W.-L. (2009). Continuous kinematic wave models of merging traffic flow. Proceedings of TRB 2009 Annual Meeting.

Jin, W. L. and Zhang, H. M. (2003a). The inhomogeneous kinematic wave traffic flow model as a resonant nonlinear system. Transportation Science, 37(3):294-311.

Jin, W.-L. and Zhang, H. M. (2003b). On the distribution schemes for determining flows through a merge. Transportation Research Part B, 37(6):521-540.

Kerner, B. S. and Konhäuser, P. (1994). Structure and parameters of clusters in traffic flow. Physical Review E, 50(1):54-83.

Klingenberg, C. and Risebro, N. (1995). Convex conservation laws with discontinuous coefficients. existence, uniqueness and asymptotic behavior. Communications in Partial Differential Equations, 20(11):1959-1990.

Lebacque, J. P. (1996). The godunov scheme and what it means for first order traffic flow models. In The International Symposium on Transportation and Traffic Theory, Lyon, France.

LeVeque, R. J. (2002). Finite volume methods for hyperbolic problems. Cambridge University Press, Cambridge; New York.

Lighthill, M. J. and Whitham, G. B. (1955). On kinematic waves: II. a theory of traffic flow on long crowded roads. Proceedings of the Royal Society of London A, 229(1178):317-345.

Lin, L., Temple, J. B., and Wang, J. (1995). A comparison of convergence rates for godunov's method and glimm's method in resonant nonlinear systems of conservation laws. SIAM Journal on Numerical Analysis, 32(3):824-840.

Mochon, S. (1987). An analysis of the traffic on highways with changing surface conditions. Math. Modelling, 9:1-11.

Nagel, K. and Schreckenberg, M. (1992). A cellular automaton model for freeway traffic. Journal de Physique I France, 2(2):2221-2229.

Osher, S. (1984). Riemann Solvers, The Entropy Condition, and Difference Approximations. SIAM Journal on Numerical Analysis, 21(2):217-235.

Osher, S. and Solomon, F. (1982). Upwind Difference Schemes for Hyperbolic Systems of Conservation Laws. Mathematics of Computation, 38(158):339-374.

Richards, P. I. (1956). Shock waves on the highway. Operations Research, 4:42-51.

Seguin, N. and Vovelle, J. (2003). Analysis and approximation of a scalar conservation law with a flux function with discontinuous coefficients. Math. Models Methods Appl. Sci, 13(2):221-257.

Smoller, J. (1983). Shock waves and reaction-diffusion equations. Springer-Verlag, New York.

van Leer, B. (1984). On the Relation Between the Upwind-Differencing Schemes of Godunov, Engquist-Osher and Roe. SIAM Journal on Scientific and Statistical Computing, 5:1.

Wong, G. C. K. and Wong, S. C. (2002). A multi-class traffic flow model: an extension of lwr model with heterogeneous drivers. Transportation Research Part A: Policy and Practice, 36(9):827841.

Zhang, P. and Liu, R. (2003). Hyperbolic conservation laws with space-dependent flux: I. Characteristics theory and Riemann problem. Journal of Computational and Applied Mathematics, 156(1):1-21.

Zhang, P. and Liu, R. (2005a). Generalization of Runge-Kutta discontinuous Galerkin method to LWR traffic flow model with inhomogeneous road conditions. Numerical Methods for Partial Differential Equations, 21(1):80-88.

Zhang, P. and Liu, R. (2005b). Hyperbolic conservation laws with space-dependent fluxes: II. General study of numerical fluxes. Journal of Computational and Applied Mathematics, 176(1):105-129.

Zhang, P., Wong, S., and Shu, C. (2006). A weighted essentially non-oscillatory numerical scheme for a multi-class traffic flow model on an inhomogeneous highway. Journal of Computational Physics, 212(2):739-756. 